\title{
Price Competition with the Attraction Demand Model: Existence of Unique Equilibrium and Its Stability
}

\author{
Guillermo Gallego, Woonghee Tim Huh \\ Industrial Engineering and Operations Research Department, Columbia University, 500 West 120th Street, \\ New York, New York 10027 \{gmg2@columbia.edu, th2113@columbia.edu\} \\ Wanmo Kang \\ Division of Applied Mathematics, Korea Advanced Institute of Science and Technology, Daejeon, 305-701, South Korea, \\ wanmokang@gmail.com \\ Robert Phillips \\ Nomis Solutions, Inc., 1111 Bayhill Drive, San Bruno, California 94066, robert.phillips@nomissolutions.com
}

\begin{abstract}
W e show the existence of Nash equilibria in a Bertrand oligopoly price competition game using a possibly asymmetric attraction demand model with convex costs under mild assumptions. We show that the equilibrium is unique and globally stable. To our knowledge, this is the first paper to show the existence of a unique equilibrium with both nonlinear demand and nonlinear costs. In addition, we guarantee the linear convergence rate of tatônnement. We illustrate the applicability of this approach with several examples arising from operational considerations that are often ignored in the economics literature.
\end{abstract}

Key words: price competition; oligopoly; attraction demand model; Nash equilibrium; convex costs History: Received: June 28, 2004; accepted: May 16, 2006.

\section{Introduction}

We consider the problem of price equilibrium in markets where multiple firms produce differentiated products. Many firms face the problem of determining pricing and inventory policies in markets where demand depends not only on their own price, but also on the prices of competing products. Section 3 lists several examples of operations management problems that fit this framework. Key questions include the existence, uniqueness, and stability of equilibrium when firms are individually setting optimal prices. We present a set of conditions under which a unique and stable pure-strategy equilibrium is guaranteed to exist in a Bertrand oligopoly price competition model when demand is determined by an attraction model and cost functions are convex. We also show that if individual firms follow a "best response approach" to current prices set by other players, then prices converge to the unique and stable pure-strategy equilibrium. Finally, we provide the convergence rate of this tatônnement scheme.

\subsection{Demand and Cost Models}

In the Bertrand oligopoly price competition model for differentiated products, a variety of demand and cost models has been used. Table 1 illustrates commonly used demand models. We let $n$ be the number of firms, which are indexed by $i=1, \ldots, n$. The demand for each firm is specified as a function of prices. Let $p_{i}$ denote the price of firm $i$, and define the price vector of competing firms by $\mathbf{p}_{-i}=$ $\left(p_{1}, \ldots, p_{i-1}, p_{i+1}, \ldots, p_{n}\right)$. Also denote the vector of all prices by $\mathbf{p}=\left(p_{1}, \ldots, p_{n}\right)=\left(p_{i}, \mathbf{p}_{-i}\right)$. The demand for each firm $i$ is given by $d_{i}=d_{i}(\mathbf{p})$. Demand functions are deterministic but can be interpreted as expected demands in many applications. We assume that firm $i$ 's demand is strictly decreasing in its price (i.e., $\left.\partial d_{i} / \partial p_{i}<0\right)$ and that products are gross substitutes (i.e., $\partial d_{i} / \partial p_{j} \geq 0$ whenever $j \neq i$ ).

In this paper we consider a generalization of the logit demand model called the attraction demand model:

$$
d_{i}(\mathbf{p}):=\frac{a_{i}\left(p_{i}\right)}{\sum_{j} a_{j}\left(p_{j}\right)+\kappa},
$$


Table 1 Common Demand Models

\begin{tabular}{lcc}
\hline Demand model & $d_{i}(\mathbf{p})$ & Restriction \\
\hline Linear & $\left(a_{i}-b_{i} p_{i}+c_{i} \sum_{j \neq i} p_{j}\right)^{+}$ & $a_{i}>0, b_{i}>0, c_{i} \geq 0$ \\
Constant & $p_{i}^{1 /(\beta-1)} /\left(\sum_{j} p_{j}^{\beta /(\beta-1)}\right)^{(1-\theta) /(1-\beta \theta)}$ & $\theta, \beta \in(0,1)$ \\
$\quad$ elasticity & & \\
Constant & $p_{i}^{-1} \cdot g\left(p_{i}\right) / \sum_{j} g\left(p_{j}\right)$ & Positive, smooth, and \\
$\quad$ expenditure & & strictly decreasing $g^{*}$ \\
Logit & $k_{i} \exp \left(\lambda p_{i}\right) / \sum_{j} k_{j} \exp \left(\lambda p_{j}\right)$ & $\lambda<0, k_{j}>0$ \\
Attraction & $a_{i}\left(p_{i}\right) /\left(\sum_{j} a_{j}\left(p_{j}\right)+\kappa\right)$ & $\kappa \geq 0$, Assumption A \\
\hline
\end{tabular}

*Examples include the CES function $g(p)=p^{-r}$ where $r>0$, and the exponential function $g(p)=\exp (-\beta p)$ where $\beta>0$.

where $\kappa$ is either 0 or strictly positive.

The attraction function $a_{i}(\cdot)$ of firm $i$ is a positive and strictly decreasing function of its price. Without any loss of generality, we normalize demand so that the total demand does not exceed 1. If $\kappa=0$, the total demand of $n$ firms equals exactly 1 ; if $\kappa$ is strictly positive, it is less than 1, possibly accounting for lost demand to an outside alternative.

Luce (1959) has shown that the attraction demand model (1) can be derived axiomatically based on simple assumptions about consumer behavior. ${ }^{1}$ As discussed in Anderson et al. (1996) and Mahajan and van Ryzin (1998), the attraction demand model has successfully been used in estimating demand in econometric studies and is increasingly accepted in marketing, e.g., Besanko et al. (1998). See So (2000), Bernstein and Federgruen (2004b), and references therein for its applications in operations management.

We now consider the cost model. We assume that cost is a function of demand alone. We denote firm $i$ 's cost function by $C_{i}\left(d_{i}\right)$ defined on $d_{i} \in[0,1]$ and assume $C_{i}$ is increasing and convex.

The profit of firm $i$ is the difference between its revenue and cost, given by

$$
\pi_{i}:=\pi_{i}(\mathbf{p}):=p_{i} \cdot d_{i}(\mathbf{p})-C_{i}\left(d_{i}(\mathbf{p})\right) .
$$

Each firm's objective is to maximize $\pi_{i}$.

In this paper, we impose mild technical conditions on the attraction demand and cost models as outlined

\footnotetext{
${ }^{1}$ Interested readers are referred to Debreu (1960) and Mahajan and van Ryzin (1998) for discussions on a paradox resulting from Luce's (1959) axioms. To avoid this paradox, the alternatives in the attraction model should be "equally dissimilar."
}

in §2. We then verify that these conditions are satisfied by commonly used attraction functions and cost models.

\subsection{Literature Review}

The study of oligopolistic interaction is a classical problem in economics. In the model proposed by Cournot (1838), firms compete on production output quantities, which in turn determine the market price. In Bertrand's (1883) model, competition is based on prices instead of production quantities. In the price competition models by Edgeworth $(1922,1925)$, each firm decides how much of its demand is satisfied, in which case a pure strategy equilibrium may or may not exist. In addition, price competition with product differentiation has been studied by Hotelling (1929), Robinson (1933), and Chamberlin (1933). An extensive treatment of the subject is found in Vives (1999). We provide a summary of results regarding the existence, uniqueness, and stability of equilibrium, followed by their application in the operations management literature.

1.2.1. Existence. There are two common methods to show existence of an equilibrium in price competition games. The first method is to obtain existence through the quasiconcavity and continuity of $\pi_{i}$ in $p_{i}$. Assuming a linear cost model, Caplin and Nalebuff (1991) show that a sufficient condition for $\pi_{i}$ to be quasiconcave is the concavity of $\log d_{i}(\mathbf{p})$ in $\log p_{i}$, which is equivalent to $\left(p_{i} / d_{i}\right)\left(\partial d_{i} / \partial p_{i}\right)$ decreasing in $p_{i}$. Another sufficient condition is the convexity of $1 / d_{i}(\mathbf{p})$ in $p_{i}$. From these conditions, the quasiconcavity of $\pi_{i}$ in $p_{i}$ holds in the logit demand model and the constant expenditure demand model with CES or exponential functions.

The second method shows existence through supermodular games. The price competition game is supermodular provided that for each $i, \pi_{i}$ is upper semicontinuous in $p_{i}$, and $\pi_{i}\left(p_{i}, \mathbf{p}_{-i}^{1}\right)-\pi_{i}\left(p_{i}, \mathbf{p}_{-i}^{2}\right)$ is increasing in $p_{i}$ whenever $\mathbf{p}_{-i}^{1} \geq \mathbf{p}_{-i}^{2}$. Topkis (1979) shows the existence of an equilibrium in supermodular games, and Milgrom and Roberts (1990) for monotone transformation of supermodular games. Thus, if the price competition game is supermodular, it has at least one equilibrium. Similarly, Milgrom and Shannon (1994) show the existence of a Nash equilibrium for a generalization of supermodular games, 
called games with strategic complementarities. Such games include instances of price competition.

Vives (1999) summarizes two additional methods of showing existence in some special cases: (i) twoplayer games with decreasing best response functions and (ii) symmetric games in which the best response function of a player depends only on the aggregate actions of others and the action sets are one dimensional. Dubey et al. (2003) use yet another approach of pseudopotential games and show existence and the convergence of a method based on fictitious plays. ${ }^{2}$ Yet they show neither the uniqueness of an equilibrium nor the convergence rate.

1.2.2. Uniqueness. The most common method to show uniqueness is the following contraction condition (see, for example, Milgrom and Roberts 1990):

$$
\frac{\partial^{2} \pi_{i}}{\partial p_{i}^{2}}+\sum_{j \neq i}\left|\frac{\partial^{2} \pi_{i}}{\partial p_{i} \partial p_{j}}\right|<0,
$$

or a similar condition in which $\pi_{i}$ is replaced by $\log \pi_{i}$. In general, it is not easy to verify this condition on the entire action space unless the demand model is symmetric. An exception is Bernstein and Federgruen (2004b), who show uniqueness using the attraction demand model and linear cost model under certain conditions. In supermodular price competition games, the contraction condition (3) is satisfied by the linear demand model with convex costs or the linear cost model and the constant elasticity, logit, or constant expenditure demand models. Thus, these sufficient conditions require either the demand model or the cost model to be linear. In fact, when the action space is unbounded, the above contraction condition (3) is always violated for some large $p_{i}{ }^{3}$

1.2.3. Stability. By definition, a set of actions at equilibrium is a fixed point of the best response mapping. A simultaneous discrete tatônnement is a sequence of actions in which the current action of each firm is the best response to the previous actions

\footnotetext{
${ }^{2} \mathrm{~A}$ fictitious play refers to the strategy in which each player uses best responses to the historical averages of opponents.

${ }^{3}$ For any fixed $\mathbf{p}_{-i}$, consider $\pi_{i}$ as we increase $p_{i}$. Because $\pi_{i}$ is strictly decreasing for large $p_{i}$ (see Proposition 2) and is bounded below (from (2)), the second derivative of $\pi_{i}$ must be positive at some point.
}

of other firms. An equilibrium is globally stable if the tatônnement converges to this equilibrium starting from any initial set of actions. Vives (1990) shows that if a supermodular game with continuous payoffs has a unique equilibrium, it is globally stable. Little is known regarding the convergence rate of the tatônnement in the price competition game.

\subsubsection{Operations Management Applications.} There is a growing interest in oligopolistic price competition in the operations literature. To predict and study market outcomes, the existence and the uniqueness of equilibrium are often required. Stability and convergence rate indicate both the robustness of equilibrium and the efficiency of computational algorithms.

Bernstein and Federgruen (2004b) study a multipleperiod inventory model with linear costs where competition is based on both price and service level. They also consider the single-period price-only competition, where the vector of service levels is given exogenously and the price vector is simultaneously determined by all sellers. This single-period competition is further described in Example 3 of §3, where demand uncertainty is multiplicative and there is neither a minimum stocking level nor a capacity constraint (i.e., $\zeta_{i}=-\infty$ and $v_{i}=\infty$ ). For a variety of demand models, including the attraction model and the linear model, an equilibrium exists and is unique. Bernstein and Federgruen (2004a) study comparative statics in pricing competition. Bernstein and Federgruen (2003) and Bernstein et al. (2002) study a supply chain in which multiple competing retailers are replenished from the single firm. In particular, Bernstein et al. (2002) show that the vendor-managed inventory arrangement can coordinate the retailers' pricing decision. All of the above models use supermodular games. Price competition in the queueing-based service systems is studied in Allon and Federgruen (2004). In their model, firms compete on price and waiting time. Their paper employs the results of this paper for the attraction demand model with convex costs.

Cachon and Harker (2002) study duopoly price competition with cost function $C_{i}\left(d_{i}\right)=c_{i}^{1} d_{i}+c_{i}^{2} d_{i}^{\gamma_{i}}$, where $c_{i}^{1}>0, c_{i}^{2} \geq 0$, and $\gamma_{i} \in[0,1)$. Note that this cost function is concave in demand. Using the linear demand model or truncated logit demand, they show 
the existence of equilibrium under certain technical conditions. Uniqueness is shown only for the symmetric case.

1.2.5. Supermodular Games and Price Competition. The existence, uniqueness, and stability of equilibrium results are easily obtained in the case of supermodular games. Yet Bernstein and Federgruen (2004b) suggest that when the game fails to be supermodular, "little can be said... in general, about the structure or cardinality of the set of Nash equilibria." Vives (1999) shows that with nonsupermodular oligopoly games, "in general, a wide array of outcomes between the monopoly and the competitive solution are possible." Consequently, it is not surprising to note that nearly all uniqueness proofs in price competition rely on the supermodularity of games and an imposed contraction assumption. Yet Vives (1999) remarks that oligopoly price competition games need not be supermodular and points out examples by Roberts and Sonnenschein (1977) and Friedman (1983). In the price competition literature, if a supermodular game has an explicit demand model and cost model, it typically assumes either the linear demand model or the linear cost model. The only exceptions are Cachon and Harker (2002), Milgrom and Shannon (1994), and Mizuno (2003). The first is restricted to symmetric duopoly. The second shows existence but not uniqueness. It does not require a convex action space, but requires a compact action space. The third shows uniqueness under a strong assumption on demand: Namely, the demand vector remains the same if the price vector is increased uniformly or multiplied by a scalar.

\subsection{Contribution and Organization}

We show the uniqueness of equilibrium in Bertrand oligopoly price competition using an attraction demand model with convex costs. We illustrate the applicability of our generalization by illustrating convex cost models that arise in inventory and service systems.

Second, the action space of most previous Bertrand oligopoly models is assumed to be compact. Thus, even if existence can be shown for the compact space, an equilibrium is possibly a boundary solution, and no interior equilibrium may exist within the compact action space. Consequently, to identify an interior equilibrium solution, an additional assumption needs to be introduced, as in Benassy (1989) and Vives (1985). In this paper, we identify sufficient conditions for the unique equilibrium to be in the interior of the set. In particular, we allow unbounded action spaces.

Finally, we show that the unique equilibrium is globally stable and guarantee a linear convergence rate of tatônnement regardless of the initial actions. This is the first paper that identifies the convergence rate of simultaneous discrete tatônnement in a Bertrand oligopoly price competition game. Because the unique equilibrium generally does not have an analytic solution, this result is useful in the numerical computation of the equilibrium.

The organization of the rest of this paper is as follows. Section 2 outlines our modeling assumptions on the attraction functions and the cost functions. This section also shows that many common attraction functions satisfy these assumptions. Section 3 lists convex cost examples in operations management, to which our model becomes applicable. Section 4 proves the existence of unique equilibrium, also shown to be globally stable in $\S 5$. Section 5 also proves the linear convergence of the tatônnement scheme. Computational results follow in $\S 6$.

\section{Assumptions}

This section lists our assumptions on the attraction function $a_{i}(\cdot)$ in (1), the profit function $\pi_{i}$ and the cost function $C_{i}(\cdot)$ in (2). We show that these assumptions are satisfied by common attraction functions.

We let $\rho_{i}:=\inf \left\{p: a_{i}(p)=0\right\}$ be the upper bound on price $p_{i}$, where $\rho_{i}$ may be infinite. Firm $i$ 's action space for price is an open interval $\left(0, \rho_{i}\right)$. Let $\mathscr{P}:=$ $\left(0, \rho_{1}\right) \times \cdots \times\left(0, \rho_{n}\right)$. Let

$$
\eta_{i}(p):=-p \cdot a_{i}^{\prime}(p) / a_{i}(p)
$$

be the elasticity of firm $i$ 's attraction function. We adopt the following simplifying notation: $f(x+):=$ $\lim _{h \downarrow x} f(h), f(x-):=\lim _{h \uparrow x} f(h), \inf \varnothing=\infty$, and $y /(y+$ $k)=1$ if $y=\infty$ and $k$ is finite.

Condition A. For each firm $i$,

(A1) $a_{i}(\cdot)$ is positive, strictly decreasing and continuously differentiable; i.e., $a_{i}(p)>0$ and $a_{i}^{\prime}(p)<0$ for all $p \in\left(0, \rho_{i}\right)$. It follows that the elasticity of attraction, $\eta_{i}(\cdot)$, is positive for $p \in\left(0, \rho_{i}\right)$. 
(A2) The elasticity $\eta_{i}(\cdot)$ is nondecreasing. In other words, when the price is higher, the percentage decrease in the attraction function per percentage change in price is also higher. This assumption is central to the existence and uniqueness proofs. It is used, for example, to show that the first-order condition (4) has a unique solution $p_{i}$ for any $\mathbf{p}_{-i}$.

(A3) If $a_{i}(0+)<\infty$, then $a_{i}^{\prime}(0+)>-\infty$. It follows that when the price is low enough, the elasticity of attraction is close to 0 ; that is, $\eta_{i}(0+)=0$. This is needed to prove the interiority of equilibria (Proposition 3); in any equilibrium, firm $i$ would never set its price $p_{i}=0$. We remark that (A3) holds vacuously if $a_{i}(0+)=\infty$.

Condition B. For each firm $i$,

(B1) $C_{i}(\cdot)$ is strictly increasing, continuously differentiable, and convex on $[0,1]$ (i.e., $c_{i}(\cdot):=C_{i}^{\prime}(\cdot)$ is positive and increasing), and satisfies $c_{i}(0+)>0$.

Condition C. For each firm $i$,

(C1) $c_{i}(0)<\rho_{i} \cdot\left(1-1 / \eta_{i}\left(\rho_{i}\right)\right)$, that is, the Lerner index $\left[p_{i}-c_{i}\left(d_{i}\right)\right] / p_{i}$ at price $p_{i}=\rho_{i}$ and demand $d_{i}=0$ is strictly larger than $1 / \eta_{i}\left(\rho_{i}\right)$. It means that no firm would set the maximum price in equilibrium because its profits are decreasing at this price. It is needed for the interiority of equilibria.

(C2) If $\kappa=0$, then $c_{i}(1)<\rho_{i}$. It means that firm $i$ could profitably serve all demand.

(C3) If $\kappa=0$, the following technical condition holds:

$$
\sum_{i=1}^{n}\left(1-\frac{1}{\eta_{i}\left(\rho_{i}\right) \cdot\left(1-c_{i}(1) / \rho_{i}\right)}\right)>1 .
$$

This inequality holds when there are at least two distinct firms $i$ satisfying $\eta_{i}\left(\rho_{i}\right)=\infty$. Note $\rho_{i}<\infty$ implies $\eta_{i}\left(\rho_{i}\right)=\infty$.

We note that both (C2) and (C3) are used for the uniqueness proof only (Proposition 6).

Proposition 1 shows that these conditions are satisfied for arbitrary cost functions satisfying Conditions (B1) and (C2):

- Generalized logit attraction function: $a_{i}(p)=$ $\exp \left(-\lambda_{i} p\right)$ with $\lambda_{i}>0$;

- Cobb-Douglas attraction function: $a_{i}(p)=p^{-\gamma_{i}}$ with $\gamma_{i}>1$;

- Linear attraction function: $a_{i}(p)=\alpha_{i}-\beta_{i} p$ with $\alpha_{i}, \beta_{i}>0$.
Proposition 1. Suppose $n \geq 2$, and cost function $C_{i}(\cdot)$ satisfies Conditions (B1) and (C2) for each $i$. Then, Conditions $\mathrm{A}, \mathrm{B}$, and $\mathrm{C}$ are satisfied, provided that each firm i's attraction function is either (i) generalized logit, (ii) CobbDouglas with an additional assumption $\gamma_{i}>2$ in case of $\kappa=0$, or (iii) linear where $\alpha_{i} / \beta_{i}>c_{i}(1)$.

Proof. (i) We get $\rho_{i}=\infty$ and $a_{i}^{\prime}(p)=-\lambda_{i} \exp \left(-\lambda_{i} p\right)$. Thus, $\eta_{i}(p)=\lambda_{i} p, \eta_{i}\left(\rho_{i}\right)=\infty, a_{i}(0+)=1$, and $a_{i}^{\prime}(0+)=$ $-\lambda_{i}$, satisfying Condition A. In addition, because $c_{i}(0)$ is finite, $\rho_{i} \cdot\left(1-1 / \eta_{i}\left(\rho_{i}\right)\right)=\infty$ implies (C1). Furthermore, $1-\left(\eta_{i}\left(\rho_{i}\right) \cdot\left(1-c_{i}(1) / \rho_{i}\right)\right)^{-1}=1$.

(ii) We have $\rho_{i}=\infty$ and $a_{i}^{\prime}(p)=-\gamma_{i} p^{-\gamma_{i}-1}$. Thus, the elasticity $\eta_{i}(p)=\gamma_{i}$ is constant for all $p \in\left(0, \rho_{i}\right)$, and $a_{i}(0+)=\infty$, satisfying Condition A. Also, (C1) follows from $\rho_{i} \cdot\left(1-1 / \eta_{i}\left(\rho_{i}\right)\right)=\rho_{i} \cdot\left(1-1 / \gamma_{i}\right)=\infty$. If $\kappa=0$, then we assume $\gamma_{i}>2$ and get $1-\left(\eta_{i}\left(\rho_{i}\right) \cdot(1-\right.$ $\left.\left.c_{i}(1) / \rho_{i}\right)\right)^{-1}=1-1 / \gamma_{i}>1 / 2$.

(iii) We have $\rho_{i}=\alpha_{i} / \beta_{i}$ and $a_{i}^{\prime}(p)=-\beta_{i}$. Thus, $\eta_{i}(p)=\beta_{i} p /\left(\alpha_{i}-\beta_{i} p\right)$, and $a_{i}(0+)=\alpha_{i}$, satisfying Condition $\mathrm{A}$. In addition, we obtain $\eta_{i}\left(\rho_{i}\right)=\infty$, and thus $\rho_{i} \cdot\left(1-1 / \eta_{i}\left(\rho_{i}\right)\right)=\rho_{i}=\alpha_{i} / \beta_{i}>c_{i}(1) \geq c_{i}(0)$, implying (C1). As $1-c_{i}(1) / \rho_{i}>0$, we obtain $1-\left(\eta_{i}\left(\rho_{i}\right) \cdot(1-\right.$ $\left.\left.c_{i}(1) / \rho_{i}\right)\right)^{-1}=1$.

Now, (C3) follows easily, because in each of the above three cases, $1-\left(\eta_{i}\left(\rho_{i}\right) \cdot\left(1-c_{i}(1) / \rho_{i}\right)\right)^{-1}$ is strictly greater than $1 / 2$.

Condition (B1) essentially means that $C_{i}(\cdot)$ is a smooth convex function in $d_{i}$. Examples of $C_{i}(\cdot)$ include the linear function and exponential function. More examples are provided in $\S 3$. We remark that attraction functions do not need to be identical. Furthermore, even the form of the attraction function may not be same among firms. Analogously, the cost functions need not have the same form either.

For the rest of this paper, we assume Conditions A, $B$, and $C$ hold. In $\$ 5$, we introduce as an additional assumption that both $C_{i}(\cdot)$ and $a_{i}(\cdot)$ are twice continuously differentiable.

\section{Examples}

In this section, we list price competition models for which the convex cost model is applicable. With the attraction demand model, the results of this paper show the existence and uniqueness of equilibrium in these models. We present some examples from inventory-capacity systems, followed by those from service systems based on queues. 


\subsection{Inventory-Capacity Systems}

Example 1. Consider the pricing problem in the stochastic inventory system with exogenously determined stocking levels. We denote stochastic demand of firm $i$ by $D_{i}(\mathbf{p})$ and its expected demand by $d_{i}(\mathbf{p})$. Demand is a function of the price vector $\mathbf{p}=\left(p_{1}, \ldots\right.$, $\left.p_{n}\right)$. We represent firm $i$ 's stochastic demand by $D_{i}\left(p_{1}, \ldots, p_{n}\right)=\varphi\left(d_{i}(\mathbf{p}), \varepsilon_{i}\right)$, where $\varepsilon_{i}$ is a random variable. (We can allow $\varphi$ to be dependent on $i$.) We suppose the continuous density function $f_{i}(\cdot)$ for $\varepsilon_{i}$ exists and let $F_{i}(\cdot)$ denote its cumulative density function.

Let $y_{i}$ be the exogenously fixed stocking level of firm $i$. For the first $y_{i}$ units, the per unit materials cost is $w_{i}$. If realized demand is at most $y_{i}$, the per unit salvage value of $w_{i}-h_{i}>0$ is obtained. Otherwise, the excess demand is met through an emergency supply at the cost of $w_{i}+b_{i}$ per unit, where $b_{i} \geq 0$. The profit of firm $i$ is the difference between its revenue and costs, and the expected profit is $\pi_{i}\left(\mathbf{p} \mid y_{i}\right)=p_{i} \cdot d_{i}(\mathbf{p})-$ $C_{i}\left(d_{i}(\mathbf{p}), y_{i}\right)$, where

$$
\begin{aligned}
C_{i}\left(d_{i}, y_{i}\right)= & w_{i} d_{i}+h_{i} E\left[y_{i}-\varphi\left(d_{i}, \varepsilon_{i}\right)\right]^{+} \\
& +b_{i} E\left[\varphi\left(d_{i}, \varepsilon_{i}\right)-y_{i}\right]^{+},
\end{aligned}
$$

and $h_{i}$ and $b_{i}$ are the per unit inventory overage and underage costs, respectively.

Our goal is to show that for fixed $y_{i}$, this function satisfies Condition (B1). We achieve this goal with two common demand uncertainty models.

- Additive demand uncertainty model: $\varphi\left(d_{i}, \varepsilon_{i}\right)=$ $d_{i}+\varepsilon_{i}$ where $E\left[\varepsilon_{i}\right]=0$. Then,

$$
\begin{aligned}
\frac{\partial C_{i}\left(d_{i}, y_{i}\right)}{\partial d_{i}} & =w_{i}-h_{i} P\left[y_{i} \geq d_{i}+\varepsilon_{i}\right]+b_{i} P\left[y_{i} \leq d_{i}+\varepsilon_{i}\right] \\
& =w_{i}-h_{i} F_{i}\left(y_{i}-d_{i}\right)+b_{i}\left(1-F_{i}\left(y_{i}-d_{i}\right)\right) .
\end{aligned}
$$

- Multiplicative demand uncertainty model: $\varphi\left(d_{i}\right.$, $\left.\varepsilon_{i}\right)=d_{i} \cdot \varepsilon_{i}$ where $\varepsilon_{i}$ is positive and $E\left[\varepsilon_{i}\right]=1$. Then,

$$
\begin{aligned}
\frac{\partial C_{i}\left(d_{i}, y_{i}\right)}{\partial d_{i}} & =w_{i}-h_{i} \int_{0}^{y_{i} / d_{i}} \varepsilon d F_{i}(\varepsilon)+b_{i} \int_{y_{i} / d_{i}}^{\infty} \varepsilon d F_{i}(\varepsilon) \\
& =w_{i}-h_{i}+\left(h_{i}+b_{i}\right) \int_{y_{i} / d_{i}}^{\infty} \varepsilon d F_{i}(\varepsilon) .
\end{aligned}
$$

In both cases, $\partial C_{i}\left(d_{i}, y_{i}\right) / \partial d_{i}$ is positive, as $w_{i}>h_{i}$ and nondecreasing in $d_{i}$. We conclude that for fixed $y_{i}$, $C_{i}\left(d_{i}, y_{i}\right)$ is strictly increasing, twice continuously differentiable, and convex in $d_{i}$. Furthermore, $\partial C_{i}\left(d_{i}, y_{i}\right) /$ $\partial d_{i}>0$ at $d_{i}=0$.
ExAmple 2. We modify Example 1 to allow the stocking decision to exist as an operational decision, where the stocking level is constrained. The sequence of events occurs as follows. (1) Firms decide prices simultaneously. (2) The price vector $\mathbf{p}=\left(p_{1}, \ldots, p_{n}\right)$ is announced. (3) Each firm $i$ decides on the stocking level $y_{i}$, subject to the minimum stocking level and capacity constraint $\zeta_{i} \leq y_{i} \leq v_{i}$. (4) For each firm $i$, demand is realized, and the appropriate overage and underage cost is incurred.

For any given $\mathbf{p}$, firm $i$ 's expected demand is given by $d_{i}=d_{i}(\mathbf{p})$, and its optimal stocking level can be computed. Let $y_{i}^{N V}\left(d_{i}\right)$ be the solution to the newsvendor problem; i.e., $P\left[\varphi\left(d_{i}, \varepsilon_{i}\right) \leq y_{i}^{N V}\left(d_{i}\right)\right]=b_{i} /\left(b_{i}+h_{i}\right)$ where $b_{i}$ and $h_{i}$ are defined in Example 1. Let $y_{i}^{*}\left(d_{i}\right)$ be the optimal stocking level, which is $y_{i}$ maximizing $\pi_{i}\left(\mathbf{p} \mid y_{i}\right)$ subject to $\zeta_{i} \leq y_{i} \leq v_{i}$. Then $y_{i}^{*}\left(d_{i}\right)$ is the point in the interval $\left[\zeta_{i}, v_{i}\right]$ that is the closest to $y_{i}^{N V}\left(d_{i}\right)$; i.e., $y_{i}^{*}\left(d_{i}\right)=\max \left\{\zeta_{i}, \min \left\{y_{i}^{N V}\left(d_{i}\right), v_{i}\right\}\right\}$.

In the additive demand uncertainty model, we get $y_{i}^{*}\left(d_{i}\right)=\max \left\{\zeta_{i}, \min \left\{d_{i}+z_{i}, v_{i}\right\}\right\}$, where $z_{i}=F_{i}^{-1}\left(b_{i} /\right.$ $\left.\left(b_{i}+h_{i}\right)\right)$. Then

$$
\begin{aligned}
& C_{i}\left(d_{i}, y_{i}^{*}\left(d_{i}\right)\right) \\
& =w_{i} d_{i}+h_{i} E\left[y_{i}^{*}\left(d_{i}\right)-\varphi\left(d_{i}, \varepsilon_{i}\right)\right]^{+}+b_{i} E\left[\varphi\left(d_{i}, \varepsilon_{i}\right)-y_{i}^{*}\left(d_{i}\right)\right]^{+} \\
& =\left\{\begin{array}{c}
w_{i} d_{i}+h_{i} E\left[\zeta_{i}-d_{i}-\varepsilon_{i}\right]^{+}+b_{i} E\left[d_{i}+\varepsilon_{i}-\zeta_{i}\right]^{+}, \\
\quad \text { if } \zeta_{i}>d_{i}+z_{i} \text { or } \zeta_{i}>v_{i} \\
w_{i} d_{i}+h_{i} E\left[z_{i}-\varepsilon_{i}\right]^{+}+b_{i} E\left[\varepsilon_{i}-z_{i}\right]^{+}, \\
\text {if } \zeta_{i} \leq d_{i}+z_{i} \leq v_{i} \\
w_{i} d_{i}+h_{i} E\left[v_{i}-d_{i}-\varepsilon_{i}\right]^{+}+b_{i} E\left[d_{i}+\varepsilon_{i}-v_{i}\right]^{+}, \\
\text {if } d_{i}+z_{i}>v_{i} \text { and } \zeta_{i} \leq v_{i} .
\end{array}\right.
\end{aligned}
$$

We can easily show that $C_{i}\left(d_{i}, y_{i}^{*}\left(d_{i}\right)\right)$ is strictly increasing, twice differentiable, and convex in $d_{i}$. Similarly, we obtain the analogous result for the multiplicative demand uncertainty model, in which $y_{i}^{*}\left(d_{i}\right)=\min \left\{d_{i} z_{i}, v_{i}\right\}$. Furthermore, if $\zeta_{i}=-\infty$ and $v_{i}=\infty$, then $C_{i}\left(d_{i}, y_{i}^{*}\left(d_{i}\right)\right)$ is linear in $d_{i}$.

ExAmple 3. We modify Example 2 to model the minimum service level requirement. Suppose that a vector $\left(f_{1}, \ldots, f_{n}\right)$ is exogenously specified such that firm $i$ 's stock-out probability should be at most $1-f_{i}$. We remove the capacity constraint (i.e., $v_{i}=\infty$ for all $i)$ to avoid infeasibility. Then, all the results in Example 2 continue to hold, with the redefinition of $z_{i}=F_{i}^{-1}\left(\max \left\{f_{i}, b_{i} /\left(b_{i}+h_{i}\right)\right\}\right)$. 
Consider a special case of this example in which the stocking levels are determined solely by the $\left(f_{1}, \ldots, f_{n}\right)$ vector (i.e., $\left.z_{i}=F_{i}^{-1}\left(f_{i}\right)\right)$, and the minimum stocking levels do not exist (i.e., $\zeta_{i}=-\infty$ ). Then $C_{i}$ is linear. In this case, the uniqueness and existence are first proven by Bernstein and Federgruen (2004b) using supermodular games.

We remark that in Examples 2 and 3, the second derivative of $C_{i}$ with respect to $d_{i}$ may not be continuous. In such cases, the existence and uniqueness still hold; yet our results regarding stability and convergence rate in $\S 5$ do not apply.

\subsection{Service Systems}

ExAmple 4. We model each firm as a single server queue with finite buffer, where the firms' buffer sizes are given exogenously. Let $\kappa_{i}$ denote the size of firm $i$ 's buffer; no more than $\kappa_{i}$ customers are allowed to the system. We assume exponential service times and the Poisson arrival process. The rate $\mu_{i}$ of service times is exogenously determined, and the rate $d_{i}$ of Poisson arrival is an output of the price competition. In the queueing theory notation, each firm $i$ is a $M / M / 1 / \kappa_{i}$ system.

We assume that the materials cost is $w_{i}>0$ per served customer, and the diverted customers' demand due to buffer overflow is met by an emergency supply at the cost of $w_{i}+b_{i}$ unit per customer, where $b_{i}>0$. The demand arrival rate $d_{i}=d_{i}(\mathbf{p})$ is determined as a function of the price vector $\mathbf{p}$. It follows that firm $i^{\prime}$ s time-average revenue is $p_{i} \cdot d_{i}-C_{i}\left(d_{i}\right)$, where $C_{i}\left(d_{i}\right)$ is the sum of $w_{i} \cdot d_{i}$ and the time-average number of customers diverted from the system is multiplied by $b_{i}$. Thus, according to elementary queueing theory (see, for example, Medhi 2003),

$$
\begin{aligned}
C_{i}\left(d_{i}\right) & =w_{i} \cdot d_{i}+b_{i} \cdot \frac{d_{i} \cdot\left(1-d_{i} / \mu_{i}\right)\left(d_{i} / \mu_{i}\right)^{\kappa_{i}}}{1-\left(d_{i} / \mu_{i}\right)^{\kappa_{i}+1}}, \quad \text { if } d_{i} \neq \mu_{i} \\
& =w_{i} \cdot d_{i}+b_{i} \cdot \frac{d_{i}}{\kappa_{i}+1}, \quad \text { if } d_{i}=\mu_{i} .
\end{aligned}
$$

Algebraic manipulation shows that $C_{i}(\cdot)$ is convex and continuously twice differentiable, satisfying $c_{i}(0)=w_{i}>0$.

ExAMPLE 5. Consider price competition among $M / D / 1$ queueing systems in which the service level $\theta_{i}$ of each firm $i$ is exogenously specified. The following sequence of events occurs. (1) Firms decide their prices simultaneously. (2) The price vector $\mathbf{p}=\left(p_{1}, \ldots, p_{n}\right)$ is announced. (3) Each firm $i$ decides on its capacity $\mu_{i}$, which is the service rate. It takes exactly $1 / \mu_{i}$ time units to serve a customer. We assume that firm $i$ 's capacity cost is proportional to its capacity. The service level is defined as the reciprocal of the expected amount of time spent in the system, and capacity should be sufficient enough to satisfy the minimum service level. (4) For each firm, customers arrive at each queueing system according to a Poisson process with rate $d_{i}$. We assume zero marginal cost of production.

The expected amount of time spent by a customer in firm $i$ 's queueing system is given by

$$
\frac{1}{\theta_{i}}=\frac{1}{\mu_{i}}+\frac{d_{i}}{2\left(\mu_{i}-d_{i}\right) \mu_{i}} .
$$

Thus, using the quadratic formula, firm i's capacity should be

$$
\mu_{i}=\frac{d_{i}}{2}+\frac{\theta_{i}}{2}+\frac{\sqrt{d_{i}^{2}+\theta_{i}^{2}}}{2}
$$

which is convex in $d_{i}$. Recall that firm i's expected profit $p_{i} \cdot d_{i}-C_{i}\left(d_{i}\right)$, where $C_{i}\left(d_{i}\right)$ is proportional to $\mu_{i}$. The cost function $C_{i}$ is convex and continuously twice differentiable. Furthermore, $C_{i}^{\prime}(0)>0$.

We remark that this simple example is a special case of a general model presented in Allon and Federgruen (2004). They provide extensive treatment of $G / G / 1$ queueing systems.

\section{Existence and Uniqueness of Equilibrium}

In this section, we show that the oligopoly price competition has a unique equilibrium. Given the price vector, each firm's profit function is given by Expression (2), where its demand is determined by (1). We first show that the first-order condition $\partial \pi_{i} / \partial p_{i}=0$ is sufficient for the Nash equilibrium (Proposition 2). For each value of a suitably defined aggregate attraction $\delta$, we show that there is at most one candidate for the solution of the first-order condition (Proposition 3). Then we demonstrate that there exists a unique value $\delta$ of the aggregator such that this candidate indeed solves the first-order condition (Propositions 5 and 6 ). We proceed by assuming Conditions A, $\mathrm{B}$, and $\mathrm{C}$. 
Let $\varsigma_{i}(\mathbf{p}):=\eta_{i}\left(p_{i}\right) \cdot\left(1-d_{i}(\mathbf{p})\right)$.

Proposition 2. Firm i's profit function $\pi_{i}$ is strictly quasiconcave in $p_{i} \in\left(0, \rho_{i}\right)$. A vector of prices $\mathbf{p}^{*}=$ $\left(p_{1}^{*}, \ldots, p_{n}^{*}\right) \in \mathscr{P}$ satisfies $\partial \pi_{i}\left(\mathbf{p}^{*}\right) / \partial p_{i}=0$ for all $i$ if and only if $\mathbf{p}^{*}$ is a Nash equilibrium in $\mathscr{P}$. Also, $p_{i}^{*}>c_{i}(0)$ for each $i$. Furthermore, the condition $\partial \pi_{i} / \partial p_{i}=0$ is equivalent to

$$
\frac{c_{i}\left(d_{i}(\mathbf{p})\right)}{p_{i}}=1-\frac{1}{\varsigma_{i}(\mathbf{p})} .
$$

Note that the condition in (4) is analogous to the inverse elasticity condition for optimal monopoly pricing.

Proof. Compute the partial derivative of $\pi_{i}$ with respect to $p_{i} \in\left(0, \rho_{i}\right)$. We abuse the prime notation and use it for the partial differentiation with respect to $p_{i}$.

$$
\begin{aligned}
\frac{\partial \pi_{i}}{\partial p_{i}} & =d_{i}^{\prime} p_{i}+d_{i}-C_{i}^{\prime}\left(d_{i}\right) d_{i}^{\prime} \\
& =\left(\frac{a_{i}}{\sum a_{j}+\kappa}\right)^{\prime}\left(p_{i}-c_{i}\left(d_{i}\right)\right)+\frac{a_{i}}{\sum a_{j}+\kappa} \\
& =\frac{a_{i}^{\prime}\left(\sum a_{j}+\kappa\right)-a_{i} a_{i}^{\prime}}{\left(\sum a_{j}+\kappa\right)^{2}}\left(p_{i}-c_{i}\left(d_{i}\right)\right)+\frac{a_{i}}{\sum a_{j}+\kappa} \\
& =\frac{a_{i}^{\prime}\left(1-d_{i}\right)}{\sum a_{j}+\kappa}\left(p_{i}-c_{i}\left(d_{i}\right)\right)+\frac{a_{i}}{\sum a_{j}+\kappa} \\
& =\left(\frac{\left(1-d_{i}\right) p_{i} a_{i}^{\prime}}{\sum a_{j}+\kappa}\right)\left(\frac{p_{i}-c_{i}\left(d_{i}\right)}{p_{i}}+\frac{a_{i}}{p_{i} a_{i}^{\prime}} \frac{1}{1-d_{i}}\right)
\end{aligned}
$$

Note that the first factor is negative. Thus, $\partial \pi_{i} / \partial p_{i}=0$ is equivalent to setting the second factor to zero, which yields (4).

Let $l_{i}\left(p_{i}\right)$ and $r_{i}\left(p_{i}\right)$ be the left and right sides of (4) respectively; that is,

$$
l_{i}\left(p_{i}\right):=\frac{c_{i}\left(d_{i}(\mathbf{p})\right)}{p_{i}} \quad \text { and } \quad r_{i}\left(p_{i}\right):=1-\frac{1}{\boldsymbol{s}_{i}(\mathbf{p})} .
$$

We claim that $r_{i}\left(p_{i}\right)-l_{i}\left(p_{i}\right)$ is a strictly increasing function of $p_{i}$. To see this, as $d_{i}(\mathbf{p})$ is strictly decreasing in $p_{i}$, by $(\mathrm{B} 1), l_{i}(\cdot)$ is strictly decreasing. Also, because $1-d_{i}(\mathbf{p})$ is positive and strictly increasing in $p_{i}$, (A2) implies that $\boldsymbol{s}_{i}(\mathbf{p})$ is positive and strictly increasing in $p_{i}$. Hence, $r_{i}(\cdot)$ is strictly increasing. Thus, the claim follows.

The first factor in (5) is negative, and the second factor is $r_{i}\left(p_{i}\right)-l_{i}\left(p_{i}\right)$, which increases strictly. Thus, $\partial \pi_{i} / \partial p_{i}$ has at most one sign change (from positive to negative), and the change occurs when $l_{i}\left(p_{i}\right)=r_{i}\left(p_{i}\right)$, or $\partial \pi_{i} / \partial p_{i}=0$. It follows that $\pi_{i}$ is strictly quasiconcave in $p_{i}$. By quasiconcavity, $\mathbf{p}^{*}=\left(p_{1}^{*}, \ldots, p_{n}^{*}\right) \in \mathscr{P}$ is a Nash equilibrium if it satisfies $\partial \pi_{i}\left(\mathbf{p}^{*}\right) / \partial p_{i}=0$ for all $i$. Because $\mathbf{p}^{*}$ is an interior solution, $\partial \pi_{i}\left(\mathbf{p}^{*}\right) / \partial p_{i} \neq 0$ for some $i$ implies that $\mathbf{p}^{*}$ is not a Nash equilibrium.

Furthermore, because $\boldsymbol{s}_{i}\left(\mathbf{p}^{*}\right)$ is positive, $\left(c_{i}\left(d_{i}\left(\mathbf{p}^{*}\right)\right)-\right.$ $\left.p_{i}^{*}\right) / p_{i}^{*}$ is negative by (4). Thus, $p_{i}^{*}>c_{i}\left(d_{i}\left(\mathbf{p}^{*}\right)\right) \geq c_{i}(0)$, where the second inequality follows from (B1).

Given a price vector, let $\delta:=\sum_{j=1}^{n} a_{j}\left(p_{j}\right)$ be the aggregate attraction. The support of $\delta$ is $\Delta:=$ $\left(0, \sum_{j=1}^{n} a_{j}(0+)\right)$. From (A1), it follows that $\delta \in \Delta$. Then $d_{i}=a_{i}\left(p_{i}\right) /(\delta+\kappa)$. Because $a_{i}^{-1}$ is well defined by (A1), we get $p_{i}=a_{i}^{-1}\left((\delta+\kappa) d_{i}\right)$. Thus, (4) is equivalent to

$$
\frac{c_{i}\left(d_{i}\right)}{a_{i}^{-1}\left((\delta+\kappa) d_{i}\right)}=1-\frac{1}{\eta_{i} \circ a_{i}^{-1}\left((\delta+\kappa) d_{i}\right) \cdot\left(1-d_{i}\right)} .
$$

Observe that there is one-to-one correspondence between $\mathbf{p}=\left(p_{1}, \ldots, p_{n}\right)$ and $\mathbf{d}=\left(d_{1}, \ldots, d_{n}\right)$, given $\delta$ (and of course, $\kappa$ ). Let $D_{i}(\delta)$ be the solution to (6) given $\delta$ (and $\kappa$ ). The existence and uniqueness of $D_{i}(\delta)$ are guaranteed by Proposition 3 below. The $D_{i}(\delta)^{\prime}$ s may not total the "correct" value of $\delta /(\delta+\kappa)$ unless a set of conditions is satisfied (Proposition 5). Proposition 6 shows the existence of a unique $\delta$ such that the $D_{i}(\delta)^{\prime}$ s sum up to $\delta /(\delta+\kappa)$.

Let $\bar{d}_{i}(\delta):=\min \left\{a_{i}(0+) /(\delta+\kappa), 1\right\}$ be an upper bound on the market share of firm $i$. For each fixed $\delta \in \Delta$, we define the following real-valued functions on $\left(0, \bar{d}_{i}(\delta)\right)$ :

$$
\begin{gathered}
L_{i}\left(x_{i} \mid \delta\right):=\frac{c_{i}\left(x_{i}\right)}{a_{i}^{-1}\left((\delta+\kappa) x_{i}\right)} \text { and } \\
R_{i}\left(x_{i} \mid \delta\right):=1-\frac{1}{\eta_{i} \circ a_{i}^{-1}\left((\delta+\kappa) x_{i}\right)\left(1-x_{i}\right)} .
\end{gathered}
$$

We remark that both $L_{i}\left(x_{i} \mid \delta\right)$ and $R_{i}\left(x_{i} \mid \delta\right)$ are continuous in $x_{i}$ in $\left(0, \bar{d}_{i}(\delta)\right)$.

Proposition 3. For each $i$ and each $\delta \in \Delta, L_{i}(\cdot \mid \delta)$ is positive and strictly increasing, and $R_{i}(\cdot \mid \delta)$ is strictly decreasing. Furthermore, $L_{i}\left(x_{i} \mid \delta\right)=R_{i}\left(x_{i} \mid \delta\right)$ has a unique solution in $\left(0, \bar{d}_{i}(\delta)\right)$; that is, $D_{i}(\delta)$ is a welldefined function of $\delta$.

Proof. Fix $\delta$. Because $a_{i}^{-1}\left((\delta+\kappa) x_{i}\right)$ is a strictly decreasing function of $x_{i}$, (B1) implies that $L_{i}\left(x_{i} \mid \delta\right)$ is positive and strictly increasing in $x_{i}$. Furthermore, because $\eta_{i} \circ a_{i}^{-1}(\cdot)$ is positive and decreasing on 
$\left(0, a_{i}(0+)\right), R_{i}\left(x_{i} \mid \delta\right)$ is strictly decreasing in $x_{i}$. Thus, $L_{i}\left(x_{i} \mid \delta\right)=R_{i}\left(x_{i} \mid \delta\right)$ has at most one solution.

From $(\mathrm{C} 1), c_{i}(0)<\rho_{i} \cdot\left(1-1 / \eta_{i}\left(\rho_{i}\right)\right)$ implies

$$
L_{i}(0+\mid \delta)=\frac{c_{i}(0)}{\rho_{i}}<1-\frac{1}{\eta_{i}\left(\rho_{i}\right)}=R_{i}(0+\mid \delta) .
$$

Now, as $x_{i} \rightarrow \bar{d}_{i}(\delta)=\min \left\{a_{i}(0+) /(\delta+\kappa), 1\right\}$, we claim that $R_{i}\left(x_{i} \mid \delta\right) \rightarrow-\infty$. To see this, consider the following two cases:

- Case $a_{i}(0+) /(\delta+\kappa)>1$ : We have $0<a_{i}^{-1}(\delta+\kappa)<$ $\rho_{i}$, which implies $0<\eta_{i} \circ a_{i}^{-1}(\delta+\kappa)<\infty$. Thus, as $x_{i} \rightarrow$ 1 , we have $1 /\left(1-x_{i}\right) \rightarrow \infty$, and $R_{i}\left(x_{i} \mid \delta\right) \rightarrow-\infty$.

- Case $a_{i}(0+) /(\delta+\kappa) \leq 1$ : Suppose $x_{i} \rightarrow a_{i}(0+) /$ $(\delta+\kappa)$. Because $a_{i}(0+)$ is finite, (A3) implies that $\eta_{i}$ 。 $a_{i}^{-1}\left((\delta+\kappa) x_{i}\right)$ approaches 0 from above. Furthermore, $1-x_{i}$ approaches a nonnegative number from above. Thus, $R_{i}\left(x_{i} \mid \delta\right) \rightarrow-\infty$.

Recall that $L_{i}\left(x_{i} \mid \delta\right)$ is positive. Thus, from the above claim, there exists at least one solution satisfying $L_{i}\left(x_{i}\right)$ $\delta)=R_{i}\left(x_{i} \mid \delta\right)$ by the intermediate value theorem.

For any aggregate attraction $\delta \in \Delta$, Proposition 3 shows that there is a unique solution $x_{i}$ satisfying $L_{i}\left(x_{i} \mid \delta\right)=R_{i}\left(x_{i} \mid \delta\right)$, and this solution is $D_{i}(\delta)$. It represents demand that maximizes firm $i$ 's profit, provided that the aggregate attraction remains at $\delta$. Also, define

$$
D(\delta):=D_{1}(\delta)+\cdots+D_{n}(\delta) .
$$

Proposition 4. All $D_{i}(\delta) s$ and $D(\delta)$ are strictly decreasing functions. Furthermore, each $D_{i}(\delta)$ is a continuous function of $\delta$.

Proof. For each $i, D_{i}(\delta)$ is a strictly decreasing function, as any increase in $\delta$ lifts the graph of $L_{i}\left(x_{i} \mid\right.$ $\delta$ ) and drops that of $R_{i}\left(x_{i} \mid \delta\right)$. For the continuity of $D_{i}$, see Appendix A.1.

Proposition 5. For fixed $\delta \in \Delta, D(\delta)=\delta /(\delta+\kappa)$ holds if and only if there exist $\mathbf{p}=\left(p_{1}, \ldots, p_{n}\right)$ and $\mathbf{d}=$ $\left(d_{1}, \ldots, d_{n}\right)$ such that the following set of conditions holds:

(i) $\delta=\sum_{j=1}^{n} a_{j}\left(p_{j}\right)$

(ii) $d_{i}=a_{i}\left(p_{i}\right) /(\delta+\kappa)$ for each $i$

(iii) $L_{i}\left(d_{i} \mid \delta\right)=R_{i}\left(d_{i} \mid \delta\right)$ for each $i$.

In this case, furthermore, the price vector corresponding to any $\delta$ satisfying $D(\delta)=\delta /(\delta+\kappa)$ is unique.

Proof. Suppose there exist $\mathbf{p} \in \mathscr{P}$ and $\mathbf{d}$ satisfying (i), (ii), and (iii). Then, by definition of $D_{i}(\delta)$ and (iii), we obtain $d_{i}=D_{i}(\delta)$, and

$$
D(\delta)=\sum_{i=1}^{n} D_{i}(\delta)=\sum_{i=1}^{n} d_{i}=\sum_{i=1}^{n} \frac{a_{i}\left(p_{i}\right)}{\delta+\kappa}=\frac{\delta}{\delta+\kappa} .
$$

Conversely, suppose $\delta$ satisfies $D(\delta)=\delta /(\delta+\kappa)$. Set $d_{i}=D_{i}(\delta)$. Then, by definition of $D_{i}(\delta)$, (iii) holds. Let $p_{i}=a_{i}^{-1}\left((\delta+\kappa) d_{i}\right)$ for each $i$, and (ii) holds. Also, (i) follows from

$$
\begin{aligned}
\sum_{j=1}^{n} a_{j}\left(p_{j}\right) & =\sum_{j=1}^{n}(\delta+\kappa) d_{j}=\sum_{j=1}^{n}(\delta+\kappa) D_{j}(\delta) \\
& =(\delta+\kappa) D(\delta)=\delta .
\end{aligned}
$$

We have established the if and only if portion and now consider the uniqueness portion. For any given $\delta$, the $L_{i}\left(d_{i} \mid \delta\right)=R_{i}\left(d_{i} \mid \delta\right)$ equation has only one solution by Proposition 3. Hence, from the one-toone correspondence between $\mathbf{p}$ and $(\delta ; \mathbf{d})$ given $\delta$, the uniqueness of $d_{i}$ implies the uniqueness of the price vector $\mathbf{p}$.

If there is $\delta \in \Delta$ satisfying $D(\delta)=\delta /(\delta+\kappa)$, then by Proposition 5, the corresponding price vector satisfies $\partial \pi_{i} / \partial p_{i}=0$ for all $i$. By Proposition 2, this price vector is a Nash equilibrium. For the unique existence of the equilibrium, it suffices to show the result of the following proposition.

Proposition 6. There exists a unique $\delta \in \Delta$ such that $D(\delta)=\delta /(\delta+\kappa)$.

Proof. There is at most one $\delta \in \Delta$ such that $D(\delta)=\delta /(\delta+\kappa)$, since $D(\delta)-\delta /(\delta+\kappa)$ is a strictly decreasing function by Proposition 4 . As each $D_{i}$ is monotone and bounded in $[0,1], \lim _{\delta \downarrow 0} D(\delta)$ in $[0,1]$ and $\lim _{\delta \uparrow \sum_{i} a_{i}(0+)} D(\delta)-\delta /(\delta+\kappa)$ exist. We claim (i) $\lim _{\delta \downarrow 0} D(\delta)-\delta /(\delta+\kappa)>0$ and (ii) $\lim _{\delta \uparrow \sum_{i} a_{i}(0+)} D(\delta)-\delta /(\delta+\kappa)<0$. From the continuity $D(\delta)-\delta /(\delta+\kappa)$, these two claims are sufficient for the result.

First we show (i). Suppose $\kappa=0$. We note that the domain of both $L_{i}$ and $R_{i}$ functions is $\left(0, \bar{d}_{i}(\delta)\right)$, and

$$
D_{i}(0+) \leq \lim _{\delta \downarrow 0} \bar{d}_{i}(\delta)=\lim _{\delta \downarrow 0} \min \left\{\frac{a_{i}(0+)}{\delta}, 1\right\}=1,
$$

where the last equality comes from $\lim _{\delta \downarrow 0} a_{i}(\delta)>0$. By rewriting Equation (6) and the continuity of all involved functions,

$$
\begin{aligned}
D_{i}(0+)= & \lim _{\delta \downarrow 0} D_{i}(\delta) \\
= & 1-\lim _{\delta \downarrow 0} \frac{1}{\eta_{i}\left(a_{i}^{-1}\left(\delta D_{i}(\delta)\right)\right)} \\
& \cdot \frac{1}{1-c_{i}\left(D_{i}(\delta)\right) / a_{i}^{-1}\left(\delta D_{i}(\delta)\right)}
\end{aligned}
$$




$$
\begin{aligned}
& =1-\frac{1}{\eta_{i}\left(a_{i}^{-1}(0+)\right)} \cdot \frac{1}{1-c_{i}\left(D_{i}(0+)\right) / a_{i}^{-1}(0+)} \\
& =1-\frac{1}{\eta_{i}\left(\rho_{i}\right)} \cdot \frac{1}{1-c_{i}\left(D_{i}(0+)\right) / \rho_{i}} \\
& \geq 1-\frac{1}{\eta_{i}\left(\rho_{i}\right)} \cdot \frac{1}{\left(1-c_{i}(1) / \rho_{i}\right)},
\end{aligned}
$$

where the last inequality follows from (B1) and (C2). Then

$$
\begin{aligned}
D(0+)-\lim _{\delta \downarrow 0} \frac{\delta}{\delta+\kappa} & =\sum_{i} D_{i}(0+)-1 \\
& \geq \sum_{i}\left(1-\frac{1}{\eta_{i}\left(\rho_{i}\right)\left(1-c_{i}(1) / \rho_{i}\right)}\right)-1,
\end{aligned}
$$

which is greater than 0 by (C3).

Suppose $\kappa>0$. We temporarily denote $L_{i}(\cdot \mid \delta)$ and $R_{i}(\cdot \mid \delta)$ by $L_{i}(\cdot \mid \delta, \kappa)$ and $R_{i}(\cdot \mid \delta, \kappa)$, respectively. Let $\alpha:=\min \left\{\kappa / 2, \sum_{i} a_{i}(0+) / 2\right\}>0$. Then,

$$
\begin{aligned}
L_{i}\left(d_{i} \mid 0+, \kappa\right) & =\frac{c_{i}\left(d_{i}\right)}{a_{i}^{-1}\left(\kappa d_{i}\right)}=L_{i}\left(d_{i} \mid \alpha, \kappa-\alpha\right) \\
R_{i}\left(d_{i} \mid 0+, \kappa\right) & =1-\frac{1}{\eta_{i} \circ a_{i}^{-1}\left(\kappa d_{i}\right)\left(1-d_{i}\right)} \\
& =R_{i}\left(d_{i} \mid \alpha, \kappa-\alpha\right) .
\end{aligned}
$$

By definition of $D_{i}(0+)$, we get $L_{i}\left(D_{i}(0+) \mid \alpha, \kappa-\alpha\right)=$ $R_{i}\left(D_{i}(0+) \mid \alpha, \kappa-\alpha\right)$. By Proposition 3, this condition implies $D_{i}(0+)>0$. Furthermore, $\delta /(\delta+\kappa) \rightarrow 0$ as $\delta \downarrow 0$. Thus, we complete the proof of claim (i).

Now we show (ii). From Equation (6) and Definition (7),

$$
\begin{aligned}
(\delta+\kappa) D_{i}(\delta) & =a_{i}\left(\frac{c_{i}\left(D_{i}(\delta)\right)}{R_{i}\left(D_{i}(\delta) \mid \delta\right)}\right)<a_{i}\left(\frac{c_{i}(0)}{R_{i}(0 \mid \delta)}\right) \\
& =a_{i}\left(\frac{c_{i}(0)}{1-1 / \eta_{i}\left(\rho_{i}\right)}\right),
\end{aligned}
$$

where the inequality follows from $(\mathrm{B} 1), D_{i}(\delta)>0$, and Proposition 3. As $c_{i}(0)$ is positive from (B1), (C1) implies $1-1 / \eta_{i}\left(\rho_{i}\right)>0$ and $0<c_{i}(0) /\left(1-1 / \eta_{i}\left(\rho_{i}\right)\right)<$ $\rho_{i}$. Because $a_{i}(\cdot)$ is strictly decreasing by (A1), we get

$$
\bar{\delta}:=\sum_{i} a_{i}\left(\frac{c_{i}(0)}{1-1 / \eta_{i}\left(\rho_{i}\right)}\right)<\sum_{i} a_{i}(0+) .
$$

Combining the above two inequalities, we have $D(\delta)=\sum_{i} D_{i}(\delta)<\bar{\delta} /(\delta+\kappa)$. Thus,

$$
\begin{aligned}
\lim _{\delta \uparrow \sum_{i} a_{i}(0+)} D(\delta) & \leq \frac{\bar{\delta}}{\sum_{i} a_{i}(0+)+\kappa}<\frac{\sum_{i} a_{i}(0+)}{\sum_{i} a_{i}(0+)+\kappa} \\
& =\lim _{\delta \uparrow \sum_{i} a_{i}(0+)} \frac{\delta}{\delta+\kappa},
\end{aligned}
$$

regardless of whether $\sum_{i} a_{i}(0+)$ is finite or infinite. ${ }^{4}$ We complete the proof of claim (ii).

THeOrem 1. There exists a unique positive pure strategy Nash equilibrium price vector $\mathbf{p}^{*} \in \mathscr{P}$. Furthermore, $\mathbf{p}^{*}$ satisfies $p_{i}^{*}>c_{i}(0)$ for all $i=1, \ldots, n$.

Proof. The result follows immediately from Propositions 5 and 6. Proposition 2 implies $p_{i}^{*}>c_{i}(0)$.

\section{Convergence of Tatônnement Scheme}

In this section, we show that the unique equilibrium is globally stable under the tatônnement scheme. Suppose each firm $i$ chooses a best-response pricing strategy: choose $p_{i}$ maximizing his profit $\pi_{i}\left(p_{1}, \ldots, p_{n}\right)$ while $p_{j}$ 's are fixed for all $j \neq i$. This section shows that the sequence of prices obtained by iterative application of this best-response strategy globally converges to the unique Nash equilibrium price vector (Theorem 2). The tatônnement convergence result not only shows the stability of the equilibrium, but also provides a computational method of finding it. We show the convergence rate is linear (Theorem 3), guaranteeing a certain degree of stability and computational efficiency.

In the tatônnement scheme we propose, a firm does not need to know the attraction functions of other firms. For the best response pricing strategy, he only needs to observe the aggregate attraction quantity of the other firms in each iteration, which can easily be deduced from its own demand and attraction value as well as $\kappa$.

In this section, we introduce additional assumptions on $C_{i}(\cdot)$ and $a_{i}(\cdot)$. We restrict them to be continuously twice differentiable to ensure the application of the implicit function theorem. This guarantees the existence of the derivatives of best-response functions. These additional assumptions are satisfied by most common cost and attraction functions.

By Theorem 1, there exists a unique equilibrium vector, which is denoted by $\mathbf{p}^{*}=\left(p_{1}^{*}, \ldots, p_{n}^{*}\right) \in \mathscr{P}$. Define $\mathbb{Q}:=\left(0, a_{1}(0+)\right) \times \cdots \times\left(0, a_{n}(0+)\right)$. Let $\mathbf{q}^{*}=$ $\left(q_{1}^{*}, \ldots, q_{n}^{*}\right) \in \mathbb{Q}$ be the corresponding attraction vector where $q_{i}^{*}:=a_{i}\left(p_{i}^{*}\right)$. Let $\hat{q}_{i}:=\sum_{j \neq i} q_{j}$ be the sum of

${ }^{4}$ If $\sum_{i} a_{i}(0+)=\infty$, then the strict inequality still holds, because $\bar{\delta}$ is always finite. 
attraction quantities of firms other than $i$. Set $\theta_{i}^{*}:=$ $q_{i}^{*} /\left(\hat{q}_{i}^{*}+\kappa\right)$ and $d_{i}^{*}:=q_{i}^{*} /\left(q_{i}^{*}+\hat{q}_{i}^{*}+\kappa\right)$, which are both positive. Suppose we fix the price $p_{j}$ for all $j \neq i$, and let $q_{i}:=a_{i}\left(p_{i}\right)$ be the corresponding attraction.

Because $a_{i}$ is one-to-one and $\delta=q_{i}+\hat{q}_{i}$, condition (6) is equivalent to

$$
\frac{c_{i}\left(q_{i} /\left(q_{i}+\hat{q}_{i}+\kappa\right)\right)}{a_{i}^{-1}\left(q_{i}\right)}=1-\frac{1}{\eta_{i} \circ a_{i}^{-1}\left(q_{i}\right)}\left(1+\frac{q_{i}}{\hat{q}_{i}+\kappa}\right) .
$$

Using an argument similar to Proposition 3 and ensuing discussion, it can be shown that there is a unique solution $q_{i}$ to (8) for each $\hat{q}_{i}$ given by any positive number less than $\sum_{j \neq i} a_{i}(0+)$ (see Appendix A.2). We call this solution $q_{i}$ the best response function $\psi_{i}\left(\hat{q}_{i}\right)$ for firm $i$. The unique equilibrium satisfies $\psi_{i}\left(\hat{q}_{i}^{*}\right)=q_{i}^{*}$ where $\hat{q}_{i}^{*}=\sum_{j \neq i} q_{j}^{*}$. Furthermore, it is easy to show that $\psi_{i}(\cdot)$ is strictly increasing. (See Appendix A.2 for the sketch of proof.)

Proposition 7. $\psi_{i}(\cdot)$ is a strictly increasing function.

From the definition of $\theta_{i}^{*}$ and $\psi_{i}\left(\hat{q}_{i}^{*}\right)=q_{i}^{*}$, we know $\psi_{i}\left(\hat{q}_{i}\right) /\left(\hat{q}_{i}+\kappa\right)=\theta_{i}^{*}$ at $\hat{q}_{i}=\hat{q}_{i}^{*}$. The following proposition characterizes the relationship between $\psi_{i}\left(\hat{q}_{i}\right) /$ $\left(\hat{q}_{i}+\kappa\right)$ and $\theta_{i}^{*}$.

Proposition 8. $\psi_{i}\left(\hat{q}_{i}\right) /\left(\hat{q}_{i}+\kappa\right)$ is strictly decreasing in $\hat{q}_{i}$ and satisfies $\psi_{i}\left(\hat{q}_{i}^{*}\right) /\left(\hat{q}_{i}^{*}+\kappa\right)=\theta_{i}^{*}$. Thus,

$$
\frac{\psi_{i}\left(\hat{q}_{i}\right)}{\hat{q}_{i}+\kappa} \begin{cases}>\theta_{i}^{*}, & \text { for } \hat{q}_{i}<\hat{q}_{i}^{*} \\ =\theta_{i}^{*}, & \text { for } \hat{q}_{i}=\hat{q}_{i}^{*} \\ <\theta_{i}^{*}, & \text { for } \hat{q}_{i}>\hat{q}_{i}^{*} .\end{cases}
$$

Furthermore, $\psi_{i}^{\prime}\left(\hat{q}_{i}\right)$ is continuous and satisfies $0<\psi_{i}^{\prime}$. $\left(\hat{q}_{i}^{*}\right)<\theta_{i}^{*}$.

Proof. From the definition $\psi_{i}\left(\hat{q}_{i}\right)$ and (8), we get

$$
\begin{aligned}
& \frac{c_{i}\left(\psi_{i}\left(\hat{q}_{i}\right) /\left(\psi_{i}\left(\hat{q}_{i}\right)+\hat{q}_{i}+\kappa\right)\right)}{a_{i}^{-1}\left(\psi_{i}\left(\hat{q}_{i}\right)\right)} \\
& \quad=1-\frac{1}{\eta_{i} \circ a_{i}^{-1} \circ \psi_{i}\left(\hat{q}_{i}\right)}\left(1+\frac{\psi_{i}\left(\hat{q}_{i}\right)}{\hat{q}_{i}+\kappa}\right) .
\end{aligned}
$$

To see the existence of $\psi_{i}^{\prime}(\cdot)$, we apply the implicit function theorem to

$$
\begin{aligned}
F(x, y)= & \frac{c_{i}(x /(x+y+\kappa))}{a_{i}^{-1}(x)}-1 \\
& +\frac{1}{\eta_{i} \circ a_{i}^{-1}(x)}\left(1+\frac{x}{y+\kappa}\right)=0
\end{aligned}
$$

with open domain, $(x, y) \in\left(0, a_{i}(0+)\right) \times\left(0, \sum_{j \neq i}\right.$. $\left.a_{i}(0+)\right)$. The Jacobian with respect to $x$ is

$$
F_{x}(x, y)=-\left(K_{1}(x, y)+J_{1}(x, y)+K_{2}(x, y)+J_{2}(x, y)\right),
$$

where

$$
\begin{aligned}
K_{1}(x, y) & :=-\frac{c_{i}^{\prime}(x /(x+y+\kappa))(y+\kappa)}{a_{i}^{-1}(x) \cdot(x+y+\kappa)^{2}}, \\
K_{2}(x, y) & :=-\frac{1}{\eta_{i} \circ a_{i}^{-1}(x) \cdot(y+\kappa)}, \\
J_{1}(x, y) & :=\frac{c_{i}(x /(x+y+\kappa))}{\left(a_{i}^{-1}(x)\right)^{2}} \cdot\left(a_{i}^{-1}\right)^{\prime}(x), \\
J_{2}(x, y) & :=\left(1+\frac{x}{y+\kappa}\right) \cdot \frac{\left(\eta_{i} \circ a_{i}^{-1}\right)^{\prime}(x)}{\left(\eta_{i} \circ a_{i}^{-1}(x)\right)^{2}} .
\end{aligned}
$$

By Conditions (A) and (B1), $c_{i}$ is positive and increasing, $a_{i}^{-1}$ is positive and strictly decreasing, and $\eta_{i}$ 。 $a_{i}^{-1}$ is positive and decreasing. Thus, both $K_{1}(x, y)+$ $K_{2}(x, y)$ and $J_{1}(x, y)+J_{2}(x, y)$ are strictly negative. Hence, $F_{x}(x, y)>0$ and the implicit function theorem confirms the existence of the continuous derivative, $\psi^{\prime}(\cdot)$, locally. Differentiating Equation (9) with respect to $\hat{q}_{i}$ yields

$$
\begin{array}{r}
-K_{1}\left(\psi_{i}\left(\hat{q}_{i}\right), \hat{q}_{i}\right) \cdot\left(\psi_{i}^{\prime}\left(\hat{q}_{i}\right)-\frac{\psi_{i}\left(\hat{q}_{i}\right)}{\hat{q}_{i}+\kappa}\right)-J_{1}\left(\psi_{i}\left(\hat{q}_{i}\right), \hat{q}_{i}\right) \cdot \psi_{i}^{\prime}\left(\hat{q}_{i}\right) \\
=K_{2}\left(\psi_{i}\left(\hat{q}_{i}\right), \hat{q}_{i}\right) \cdot\left(\psi_{i}^{\prime}\left(\hat{q}_{i}\right)-\frac{\psi_{i}\left(\hat{q}_{i}\right)}{\hat{q}_{i}+\kappa}\right)+J_{2}\left(\psi_{i}\left(\hat{q}_{i}\right), \hat{q}_{i}\right) \cdot \psi_{i}^{\prime}\left(\hat{q}_{i}\right) .
\end{array}
$$

Thus,

$$
\begin{aligned}
\psi_{i}^{\prime}\left(\hat{q}_{i}\right)= & \left(K_{1}\left(\psi_{i}\left(\hat{q}_{i}\right), \hat{q}_{i}\right)+K_{2}\left(\psi_{i}\left(\hat{q}_{i}\right), \hat{q}_{i}\right)\right) \\
& \cdot\left(J_{1}\left(\psi_{i}\left(\hat{q}_{i}\right), \hat{q}_{i}\right)+J_{2}\left(\psi_{i}\left(\hat{q}_{i}\right), \hat{q}_{i}\right)+K_{1}\left(\psi_{i}\left(\hat{q}_{i}\right), \hat{q}_{i}\right)\right. \\
& \left.\quad+K_{2}\left(\psi_{i}\left(\hat{q}_{i}\right), \hat{q}_{i}\right)\right)^{-1} \cdot \frac{\psi_{i}\left(\hat{q}_{i}\right)}{\hat{q}_{i}+\kappa}<\frac{\psi_{i}\left(\hat{q}_{i}\right)}{\hat{q}_{i}+\kappa},
\end{aligned}
$$

and it follows

$$
\frac{d}{d \hat{q}_{i}}\left(\frac{\psi_{i}\left(\hat{q}_{i}\right)}{\hat{q}_{i}+\kappa}\right)=\frac{\psi_{i}^{\prime}\left(\hat{q}_{i}\right)\left(\hat{q}_{i}+\kappa\right)-\psi_{i}\left(\hat{q}_{i}\right)}{\left(\hat{q}_{i}+\kappa\right)^{2}}<0
$$

Furthermore, (10) implies $\psi_{i}^{\prime}\left(\hat{q}_{i}\right)$ is continuous, and $\theta_{i}^{*}>\psi_{i}^{\prime}\left(\hat{q}_{i}^{*}\right)$ follows from the definition of $\theta_{i}^{*}$ and $\psi_{i}\left(\hat{q}_{i}^{*}\right)=q_{i}^{*}$. Proposition 7 implies $\theta_{i}^{*}>0$.

Let $\mathbf{q}=\left(q_{1}, \ldots, q_{n}\right)$. We denote the vector of best response functions by $\Psi(\mathbf{q})=\left(\psi_{1}\left(\hat{q}_{1}\right), \ldots, \psi_{n}\left(\hat{q}_{n}\right)\right) \in \mathbb{Q}$, where $\hat{q}_{i}=\sum_{j \neq i} q_{j}$. Note that $\mathbf{q}^{*}=\left(q_{1}^{*}, \ldots, q_{n}^{*}\right)$ is a fixed point of $\Psi$; i.e., $\Psi\left(\mathbf{q}^{*}\right)=\mathbf{q}^{*}$. By Proposition 7 , we have $\Psi\left(\mathbf{q}^{1}\right)<\Psi\left(\mathbf{q}^{2}\right)$ whenever two vectors $\mathbf{q}^{1}$ and $\mathbf{q}^{2}$ satisfy $\mathbf{q}^{1}<\mathbf{q}^{2}$. (The inequalities are componentwise.) 
We now show that best-response pricing converges to the unique equilibrium. We define the sequence $\left\{\mathbf{q}^{(0)}, \mathbf{q}^{(1)}, \mathbf{q}^{(2)}, \ldots\right\} \subset \mathbb{Q}$ by $\mathbf{q}^{(k+1)}:=\Psi\left(\mathbf{q}^{(k)}\right)$ for $k \geq 0$.

THEOREM 2. If each firm employs the best response strategy based on the prices of other firms in the previous iteration, the sequence of price vectors converges to the unique equilibrium price vector.

Proof. Let $\mathbf{q}^{(0)} \in \mathbb{Q}$ denote the attraction vector associated with the initial price vector. Choose $\underline{\mathbf{q}}^{(0)}, \overline{\mathbf{q}}^{(0)} \in \mathbb{Q}$ such that $\underline{\mathbf{q}}^{(0)}<\mathbf{q}^{(0)}<\overline{\mathbf{q}}^{(0)}$ and $\underline{\mathbf{q}}^{(0)}<\mathbf{q}^{*}<$ $\overline{\overline{\mathbf{q}}}^{(0)}$. Such $\underline{\mathbf{q}}^{(0)}$ and $\overline{\mathbf{q}}^{(0)}$ exist because $\mathbb{Q}$ is a box-shaped open set.

For each $k \geq 0$, we define $\underline{\mathbf{q}}^{(k+1)}:=\Psi\left(\underline{\mathbf{q}}^{(k)}\right)$ and $\overline{\mathbf{q}}^{(k+1)}:=\Psi\left(\overline{\mathbf{q}}^{(k)}\right)$. From the monotonicity of $\Psi(\cdot)$ (Proposition 7) and $\Psi\left(\mathbf{q}^{*}\right)=\mathbf{q}^{*}$, we get

$$
\underline{\mathbf{q}}^{(k)}<\mathbf{q}^{(k)}<\overline{\mathbf{q}}^{(k)} \text { and } \underline{\mathbf{q}}^{(k)}<\mathbf{q}^{*}<\overline{\mathbf{q}}^{(k)} .
$$

Let $u^{(k)}:=\max _{i}\left\{\hat{\bar{q}}_{i}^{(k)} / \hat{q}_{i}^{*}\right\}$. Clearly, $u^{(k)}>1$ for all $k$ by (12). We show that the sequence $\left\{u^{(k)}\right\}_{k=0}^{\infty}$ is strictly decreasing. For each $i$,

$$
\begin{aligned}
\bar{q}_{i}^{(k+1)} & =\psi_{i}\left(\hat{\bar{q}}_{i}^{(k)}\right)<\left(\hat{\bar{q}}_{i}^{(k)}+\kappa\right) \cdot \theta_{i}^{*} \\
& =\left(\hat{\bar{q}}_{i}^{(k)}+\kappa\right) \cdot \frac{q_{i}^{*}}{\hat{q}_{i}^{*}+\kappa} \leq \frac{\hat{\bar{q}}_{i}^{(k)}}{\hat{q}_{i}^{*}} \cdot q_{i}^{*} \leq u^{(k)} q_{i}^{*},
\end{aligned}
$$

where the first inequality comes from Proposition 8, the second one from (12), and the last one from the definition of $u^{(k)}$. Thus,

$$
\hat{\bar{q}}_{i}^{(k+1)}=\sum_{j \neq i} \bar{q}_{j}^{(k+1)}<\sum_{j \neq i} u^{(k)} q_{j}^{*}=u^{(k)} \hat{q}_{i}^{*},
$$

and $u^{(k+1)}=\max _{i}\left\{\hat{\bar{q}}_{i}^{(k+1)} / \hat{q}_{i}^{*}\right\}<u^{(k)}$.

As $\left\{u^{(k)}\right\}_{k=0}^{\infty}$ is a monotone and bounded sequence, it converges. Let $u^{\infty}:=\lim _{k \rightarrow \infty} u^{(k)}$. We claim $u^{\infty}=1$. Suppose, by way of contradiction, that $u^{\infty}>1$. By Proposition $8, \psi_{i}\left(\hat{q}_{i}\right) /\left(\hat{q}_{i}+\kappa\right)$ is strictly decreasing in $\hat{q}_{i}$. Thus, for any $\hat{q}_{i} \geq \frac{1}{2}\left(1+u^{\infty}\right) \cdot \hat{q}_{i}^{*}$, there exists $\epsilon \in(0,1)$ such that for each $i$, we have

$$
\frac{\psi_{i}\left(\hat{q}_{i}\right)}{\left(\hat{q}_{i}+\kappa\right)} \leq(1-\epsilon) \cdot \frac{\psi_{i}\left(\hat{q}_{i}^{*}\right)}{\hat{q}_{i}^{*}+\kappa}=(1-\epsilon) \cdot \frac{q_{i}^{*}}{\hat{q}_{i}^{*}+\kappa} .
$$

For any $k$, if $\hat{\bar{q}}_{i}^{(k)} \geq \frac{1}{2}\left(1+u^{\infty}\right) \cdot \hat{q}_{i}^{*}$, then

$$
\begin{aligned}
\bar{q}_{i}^{(k+1)} & =\psi_{i}\left(\hat{\bar{q}}_{i}^{(k)}\right) \leq\left(\hat{\bar{q}}_{i}^{(k)}+\kappa\right) \cdot(1-\epsilon) \cdot \frac{q_{i}^{*}}{\hat{q}_{i}^{*}+\kappa} \\
& \leq \frac{\hat{\bar{q}}_{i}^{(k)}}{\hat{q}_{i}^{*}} \cdot q_{i}^{*} \cdot(1-\epsilon) \leq(1-\epsilon) \cdot u^{(k)} \cdot q_{i}^{*} .
\end{aligned}
$$

Otherwise, we have $\hat{q}_{i}^{*}<\hat{\bar{q}}_{i}^{(k)}<\frac{1}{2}\left(1+u^{\infty}\right) \cdot \hat{q}_{i}^{*}$. By Proposition 8

$$
\begin{aligned}
\bar{q}_{i}^{(k+1)} & =\psi_{i}\left(\hat{\bar{q}}_{i}^{(k)}\right)<\left(\hat{\bar{q}}_{i}^{(k)}+\kappa\right) \cdot \frac{q_{i}^{*}}{\hat{q}_{i}^{*}+\kappa} \\
& \leq \frac{\hat{\bar{q}}_{i}^{(k)}}{\hat{q}_{i}^{*}} \cdot q_{i}^{*} \leq \frac{1}{2}\left(1+u^{\infty}\right) \cdot q_{i}^{*} .
\end{aligned}
$$

Therefore, we conclude, using an argument similar to (13),

$$
u^{(k+1)} \leq \max \left\{(1-\epsilon) \cdot u^{(k)},\left(1+u^{\infty}\right) / 2\right\} .
$$

From $u^{(k+1)}>u^{\infty}>\left(1+u^{\infty}\right) / 2$, we obtain $u^{(k+1)} \leq(1-$ $\epsilon) \cdot u^{(k)}$, implying $u^{(k)} \rightarrow 1$ as $k \rightarrow \infty$. This is a contradiction.

Similarly, we can show that $l^{(k)}:=\min _{i}\left\{\hat{\underline{q}}_{i}^{(k)} / \hat{q}_{i}^{*}\right\}$ is a strictly increasing sequence converging to $\overline{1}^{i}$.

The following corollary identifies a sufficient condition for the monotonicity of the sequence of attraction vectors (and also for price vectors).

Corollary 1. If $\mathbf{q}^{(0)}>\Psi\left(\mathbf{q}^{(0)}\right)$ or $\mathbf{q}^{(0)}<\Psi\left(\mathbf{q}^{(0)}\right)$, then $\left\{\mathbf{q}^{(k)}\right\}_{k \geq 0}$ is monotone.

Proof. Suppose $\mathbf{q}^{(0)}>\Psi\left(\mathbf{q}^{(0)}\right)=\mathbf{q}^{(1)}$. We proceed by induction. Assume that $\mathbf{q}^{(j)}>\mathbf{q}^{(j+1)}$ for all $j<k$. Then, $\mathbf{q}^{(k)}-\mathbf{q}^{(k+1)}=\Psi\left(\mathbf{q}^{(k-1)}\right)-\Psi\left(\mathbf{q}^{(k)}\right)>\mathbf{0}$ because $\Psi(\cdot)$ is strictly increasing and $\mathbf{q}^{(k-1)}>\mathbf{q}^{(k)}$.

The following proposition shows the linear convergence of tatônnement in the space of attraction values.

Proposition 9. The sequence $\left\{\mathbf{q}^{(k)}\right\}_{k \geq 0}$ converges linearly.

Proof. Consider $\left\{\mathbf{q}^{(k)}\right\}_{k=0}^{\infty}$ and $\left\{\overline{\mathbf{q}}^{(k)}\right\}_{k=0}^{\infty}$ in the proof of Theorem 2. Recall $\underline{\mathbf{q}}^{(k)}<\mathbf{q}^{(k)}<\overline{\mathbf{q}}^{(k)}$ and $\underline{\mathbf{q}}^{(k)}<\mathbf{q}^{*}<$ $\overline{\mathbf{q}}^{(k)}$. We will show that $\mathbf{q}^{(k)}$ and $\overline{\mathbf{q}}^{(k)}$ converges to $\mathbf{q}^{*}$ linearly. As $\mathbb{Q}$ is a box-shaped open set, there exists a convex compact set $\mathscr{B} \subset \mathbb{Q}$ containing all elements of $\left\{\underline{\mathbf{q}}^{(k)}\right\}_{k=0}^{\infty}$ and $\left\{\overline{\mathbf{q}}^{(k)}\right\}_{k=0}^{\infty}$. From (11) in the proof of Proposition 8 , there exists $\delta>0$ such that for any $\mathbf{q} \in \mathscr{B}$, we have

$$
\frac{d}{d \hat{q}_{i}}\left(\frac{\psi\left(\hat{q}_{i}\right)}{\hat{q}_{i}+\kappa}\right) \leq-\delta .
$$

From integrating both sides of the above expression from $\hat{q}_{i}^{*}$ to $\hat{\bar{q}}_{i}^{(k)}$,

$$
\frac{\psi_{i}\left(\hat{\bar{q}}_{i}^{(k)}\right)}{\hat{\bar{q}}_{i}^{(k)}+\kappa}-\frac{\psi\left(\hat{q}_{i}^{*}\right)}{\hat{q}_{i}^{*}+\kappa} \leq-\delta\left(\hat{\bar{q}}_{i}^{(k)}-\hat{q}_{i}^{*}\right),
$$

as the line segment connecting $\mathbf{q}^{*}$ and $\overline{\mathbf{q}}^{(k)}$ lies within $\mathscr{B}$. 
Gallego et al.: Price Competition with the Attraction Demand Model

Table 2 Tatônnement Computation Output/Generalized Logit Attraction Model

\begin{tabular}{lccrr}
\hline$\kappa$ & \multicolumn{1}{c}{$\mathbf{p}^{*}$} & $\mathbf{d}^{*}$ & $\sum_{i} d_{i}^{*}$ & Iter \\
\hline 0 & $(3.6987,2.5936,2.0659,1.7702,1.5826)$ & $(0.2499,0.2271,0.2013,0.1738,0.1479)$ & 1 & 14 \\
0.01 & $(3.6807,2.5837,2.0602,1.7666,1.5801)$ & $(0.2467,0.2238,0.1980,0.1708,0.1452)$ & 0.9844 & 14 \\
0.05 & $(3.6170,2.5490,2.0401,1.7538,1.5714)$ & $(0.2348,0.2118,0.1862,0.1600,0.1356)$ & 0.9283 & 13 \\
0.1 & $(3.5512,2.5137,2.0198,1.7409,1.5626)$ & $(0.2219,0.1989,0.1738,0.1487,0.1257)$ & 0.8689 & 12 \\
0.5 & $(3.2722,2.3687,1.9366,1.6879,1.5265)$ & $(0.1582,0.1375,0.1172,0.0985,0.0823)$ & 0.5937 & 9 \\
\hline
\end{tabular}

Notes. $\left(\lambda_{1}, \ldots, \lambda_{5}\right)=(0.5,0.75,1,1.25,1.5) .\left(y_{1}, \ldots, y_{5}\right)=(0.5,0.6,0.7,0.8,0.9)$.

Table 3 Tatônnement Computation Output/Generalized Logit Attraction Model

\begin{tabular}{lccrr}
\hline$\kappa$ & \multicolumn{1}{c}{$\mathbf{p}^{*}$} & $\mathbf{d}^{*}$ & $\sum_{i} d_{i}^{*}$ & Iter \\
\hline 0 & $(1.6427,1.7932,2.0625,2.5429,3.5335)$ & $(0.1334,0.1666,0.1993,0.2328,0.2679)$ & 1 & 14 \\
0.01 & $(1.6391,1.7890,2.0569,2.5347,3.5192)$ & $(0.1313,0.1639,0.1961,0.2292,0.2641)$ & 0.9846 & 14 \\
0.05 & $(1.6264,1.7743,2.0376,2.5055,3.4686)$ & $(0.1236,0.1542,0.1847,0.2164,0.2502)$ & 0.9291 & 13 \\
0.1 & $(1.6136,1.7594,2.0179,2.4757,3.4164)$ & $(0.1154,0.1440,0.1726,0.2028,0.2353)$ & 0.8701 & 12 \\
0.5 & $(1.5613,1.6989,1.9362,2.3498,3.1930)$ & $(0.0779,0.0969,0.1169,0.1391,0.1642)$ & 0.5948 & 9
\end{tabular}

Notes. $\left(\lambda_{1}, \ldots, \lambda_{5}\right)=(1.5,1.25,1,0.75,0.5) .\left(y_{1}, \ldots, y_{5}\right)=(0.5,0.6,0.7,0.8,0.9)$.

Define $\delta_{1}:=\delta \cdot \min _{i} \min _{\mathbf{q} \in \mathscr{F}}\left\{\left(\hat{q}_{i}+\kappa\right) \cdot \hat{q}_{i}^{*} / q_{i}^{*}\right\}>0$. We choose $\delta>0$ as sufficiently small such that $\delta_{1}<1$. Recall $\bar{q}_{i}^{(k+1)}=\psi_{i}\left(\hat{\bar{q}}_{i}^{(k)}\right)$ and $q_{i}^{*}=\psi\left(\hat{q}_{i}^{*}\right)$. Rearranging the above inequality and multiplying it by $\left(\hat{\bar{q}}_{i}^{(k)}+\kappa\right) / q_{i}^{*}$,

$$
\begin{aligned}
\bar{q}_{i}^{(k+1)} / q_{i}^{*} & \leq\left(\hat{\bar{q}}_{i}^{(k)}+\kappa\right) /\left(\hat{q}_{i}^{*}+\kappa\right)-\delta \cdot\left(\hat{\bar{q}}_{i}^{(k)}-\hat{q}_{i}^{*}\right) \cdot\left(\hat{\bar{q}}_{i}^{(k)}+\kappa\right) / q_{i}^{*} \\
& \leq \hat{\bar{q}}_{i}^{(k)} / \hat{q}_{i}^{*}-\delta_{1} \cdot\left(\hat{\bar{q}}_{i}^{(k)} / \hat{q}_{i}^{*}-1\right) \\
& =\left(1-\delta_{1}\right) \cdot \hat{\bar{q}}_{i}^{(k)} / \hat{q}_{i}^{*}+\delta_{1},
\end{aligned}
$$

where the second inequality comes from $\hat{\bar{q}}_{i}^{(k)}>\hat{q}_{i}^{*}$ and the definition of $\delta_{1}$.

Let $\rho(k):=\max _{i}\left\{\bar{q}_{i}^{(k)} / q_{i}^{*}\right\}$. Thus, $\bar{q}_{j}^{(k)} \leq \rho(k) \cdot q_{j}^{*}$ holds for all $j$, and summing this inequality for all $j \neq i$, we get $\hat{\bar{q}}_{i}^{(k)} \leq \rho(k) \cdot \hat{q}_{i}^{*}$. Thus, $\bar{q}_{i}^{(k+1)} / q_{i}^{*}$ is bounded above by $\left(1-\delta_{1}\right) \cdot \rho(k)+\delta_{1}$ for each $i$, and we obtain

$$
\rho(k+1) \leq\left(1-\delta_{1}\right) \cdot \rho(k)+\delta_{1} .
$$

Using induction, it is easy to show

$$
\rho(k) \leq\left(1-\delta_{1}\right)^{k} \cdot(\rho(0)-1)+1 .
$$

Therefore, we obtain

$$
\begin{aligned}
\max _{i}\left\{\left(\bar{q}_{i}^{(k)}-q_{i}^{*}\right) / q_{i}^{*}\right\} & =\rho(k)-1 \leq\left(1-\delta_{1}\right)^{k} \cdot(\rho(0)-1) \\
& =\left(1-\delta_{1}\right)^{k} \cdot \max _{i}\left\{\left(\bar{q}_{i}^{(0)}-q_{i}^{*}\right) / q_{i}^{*}\right\}
\end{aligned}
$$

and

$$
\max _{i}\left\{\bar{q}_{i}^{(k)}-q_{i}^{*}\right\} \leq\left(1-\delta_{1}\right)^{k} \cdot \max _{i}\left\{q_{i}^{*}\right\} \cdot \max _{i}\left\{\left(\bar{q}_{i}^{(0)}-q_{i}^{*}\right) / q_{i}^{*}\right\},
$$

showing the linear convergence of the upper bound sequence $\overline{\mathbf{q}}^{(k)}$ (Bertsekas 1995). A similar argument shows the linear convergence of $\mathbf{q}^{(k)}$.

The linear convergence in the above proposition is not with respect to the price vector but with respect to the attraction vector, i.e., not in $\mathbf{p}$ but in $\mathbf{q}$. Yet the following theorem also shows the linear convergence with respect to the price vector. Let $\left\{\mathbf{p}^{(k)}\right\}_{k \geq 0}$ be the sequence defined by $p_{i}^{(k)}:=a_{i}^{-1}\left(q_{i}^{(k)}\right)$.

Theorem 3. The sequence $\left\{\mathbf{p}^{(k)}\right\}_{k \geq 0}$ converges linearly.

Proof. Consider $\left\{\underline{\mathbf{q}}^{(k)}\right\}_{k=0}^{\infty}$ and $\left\{\overline{\mathbf{q}}^{(k)}\right\}_{k=0}^{\infty}$ in the proof of Proposition 9. Let $\left\{\underline{\mathbf{p}}^{(k)}\right\}_{k=0}^{\infty}$ and $\left\{\overline{\mathbf{p}}^{(k)}\right\}_{k=0}^{\infty}$ be the corresponding sequences of price vectors. Within the compact interval $\left[\bar{p}_{i}^{(0)}, p_{i}^{(0)}\right]$, the derivative of $a_{i}$ is continuous and its infimum is strictly negative. By the inverse function theorem, the derivative of $a_{i}^{-1}(\cdot)$ is continuous in the compact domain of $\left[q_{i}^{(0)}, \bar{q}_{i}^{(0)}\right]$. Recall $p_{i}^{*}=a_{i}^{-1}\left(q_{i}^{*}\right)$. There exists some bound $\vec{M}^{-}>0$ such that

$$
\left|p_{i}-p_{i}^{*}\right|=\left|a_{i}^{-1}\left(q_{i}\right)-a_{i}^{-1}\left(q_{i}^{*}\right)\right| \leq M \cdot\left|q_{i}-q_{i}^{*}\right|
$$

whenever $a_{i}\left(p_{i}\right)=q_{i} \in\left[q_{i}^{(0)}, \bar{q}_{i}^{(0)}\right]$ for all $i$. From the proof of Proposition 9, we get $q_{i}^{(k)} \in\left(\underline{q}_{i}^{(k)}, \bar{q}_{i}^{(k)}\right) \subset\left[\underline{q}_{i}^{(0)}\right.$, $\left.\bar{q}_{i}^{(0)}\right]$. Therefore, the linear convergence of $\left\{\mathbf{q}^{(k)}\right\}_{k \geq 0}$ implies the linear convergence of $\left\{\mathbf{p}^{(k)}\right\}_{k \geq 0}$. 


\begin{tabular}{rcc}
\hline$n$ & $\left(\lambda_{1}, \ldots, \lambda_{n}\right)$ & $\left(y_{1}, \ldots, y_{n}\right)$ \\
\hline 2 & $(0.5,1.5)$ & $(0.5,0.9)$ \\
5 & $(0.5,0.75,1,1.25,1.5)$ & $(0.5,0.6,0.7,0.8,0.9)$ \\
10 & $(0.5,0.6,0.7,0.8,0.9,1.1,1.2,1.3,1.4,1.5)$ & $(0.45,0.5,0.55,0.6,0.65,0.7,0.75,0.8,0.85,0.9)$ \\
\hline
\end{tabular}

Note. Input data for Figure 2

\section{Numerical Results}

In this section, we perform the computation of the tatônnement scheme using Example 1 and report on its convergence behavior.

We first consider the price competition among $n=5$ firms. We use generalized logit attraction functions.

Figure 1 Generalized Logit Attraction Model/Initialization $\mathbf{p}^{(0)}=$ $\left(C_{1}^{\prime}(0), \ldots, C_{n}^{\prime}(0)\right) \cdot n=5$

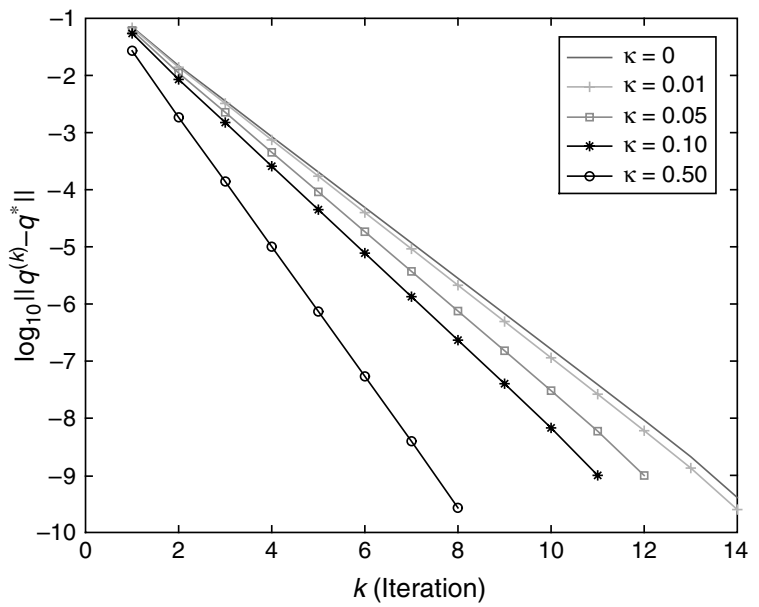

(a) Convergence of $\mathbf{q}^{(k)}$

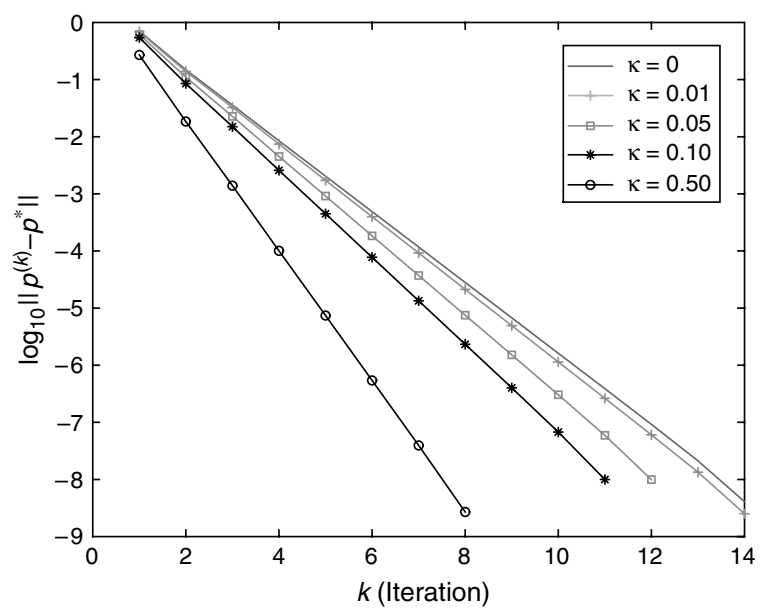

(b) Convergence of $\mathbf{p}^{(k)}$
Thus,

$$
d_{i}(\mathbf{p})=\frac{\exp \left(-\lambda_{i} p_{i}\right)}{\sum_{j=1}^{n} \exp \left(-\lambda_{j} p_{j}\right)+\kappa}
$$

where the parameter $\lambda_{i}>0$ depends on each firm $i$. We used $\kappa=0,0.01,0.05,0.1,0.5$ values. For stochastic demand, we use the additive demand uncertainty model $\delta\left(d_{i}, \varepsilon\right)=d_{i}+\varepsilon_{i}$, where $\varepsilon_{i}$ has a Gaussian dis-

Figure 2 Generalized Logit Attraction Model/Initialization $\mathbf{p}^{(0)}=$ $\left(C_{1}^{\prime}(0), \ldots, C_{n}^{\prime}(0)\right) . \kappa=0.01$.

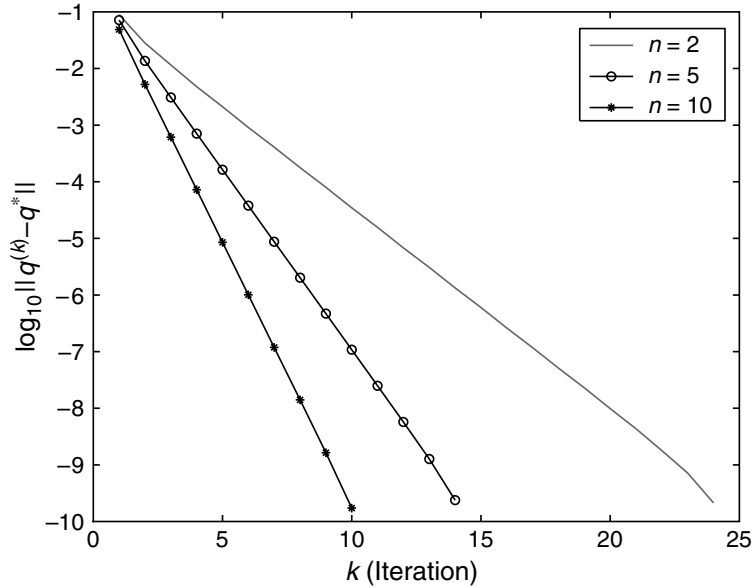

(a) Convergence of $\mathbf{q}^{(k)}$

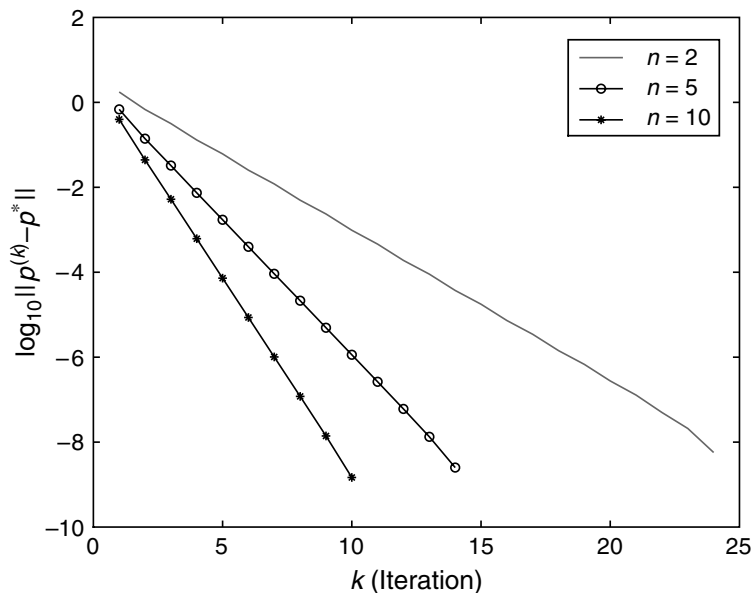

(b) Convergence of $\mathbf{p}^{(k)}$ 
tribution with mean 0 and variance 0.2 . The cost function in Example 1 is given by

$$
C_{i}^{\prime}\left(d_{i}\right)=w_{i}-h_{i} P\left(y_{i} \geq d_{i}+\varepsilon_{i}\right)+b_{i} P\left(y_{i} \leq d_{i}+\varepsilon_{i}\right),
$$

where $w_{i}=1.0, h_{i}=0.2$, and $b_{i}=2.0$ for each firm $i$. The value of $y_{i}$ depends on firm $i$. We initialize the algorithm with $\mathbf{p}^{(0)}=\left(C_{1}^{\prime}(0), \ldots, C_{n}^{\prime}(0)\right)$. The algorithm is terminated when the Euclidean distance between two successive $\mathbf{p}^{(k)}$ vectors is less than tolerance $10^{-8}$. We use Matlab 5.3 on a PC notebook with 900 megahertz processor speed and 384 megabytes of memory. A typical iteration takes several seconds, most

\section{Figure 3 Cobb-Douglas Attraction Model}

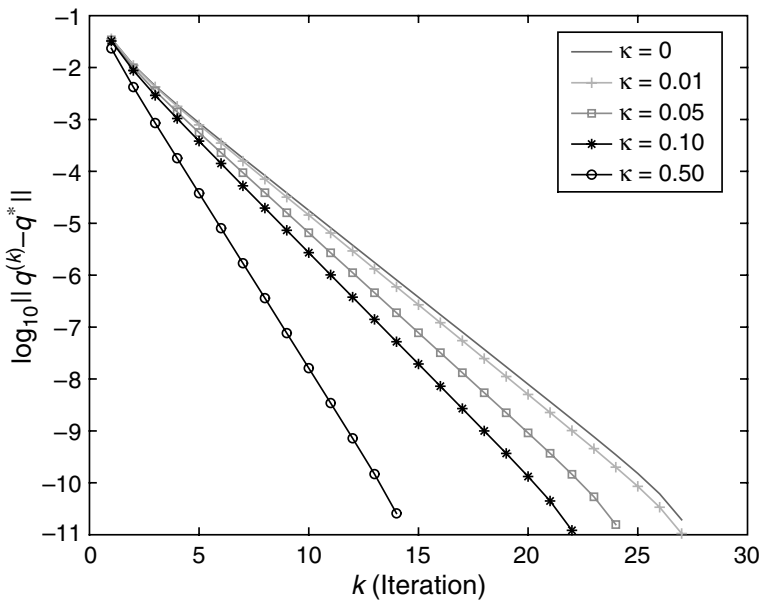

(a) Convergence of $\mathbf{q}^{(k)}$

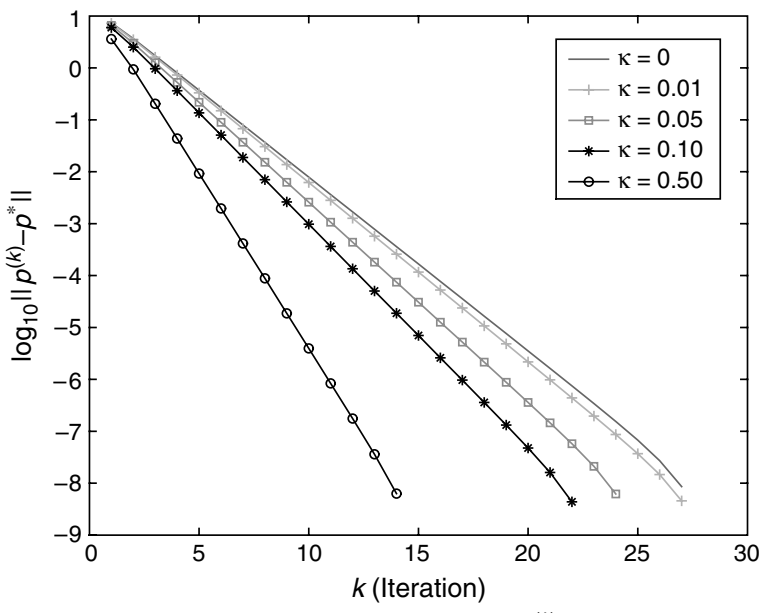

(b) Convergence of $\mathbf{p}^{(k)}$

Notes. $d_{i}(\mathbf{p})=\left(p_{i}^{-\gamma_{i}}\right) /\left(\sum_{j=1}^{n} p_{j}^{-\gamma_{j}}+\kappa\right)$, where $\gamma=(1.1,1.6,2.1,2.6,3.1)$. Initialization $\mathbf{p}^{(0)}=\left(C_{1}^{\prime}(0), \ldots, C_{n}^{\prime}(0)\right) . n=5$. of which is spent invoking the Gaussian cumulative density function in (14).

Table 2 shows the equilibrium price vector $\mathbf{p}^{*}$ and expected demand vector $\mathbf{d}^{*}$ provided $\left(\lambda_{1}, \ldots, \lambda_{5}\right)=$ $(0.5,0.75,1,1.25,1.5)$ and $\left(y_{1}, \ldots, y_{5}\right)=(0.5,0.6,0.7$, $0.8,0.9)$. The column marked by $\sum_{i} d_{i}^{*}$ is the total expected demand as a function of $\kappa$. The last column shows the number of iterations until termination. We repeat computation with $\left(\lambda_{1}, \ldots, \lambda_{5}\right)=$ $(1.5,1.25,1,0.75,0.5)$, and report results in Table 3.

Using the same $\lambda$ vector and $y$ vector as in Table 2, Figure 1 plots $\left\|\mathbf{q}^{(k)}-\mathbf{q}^{*}\right\|$ as a function of iteration

\section{Figure 4 Linear Attraction Model}

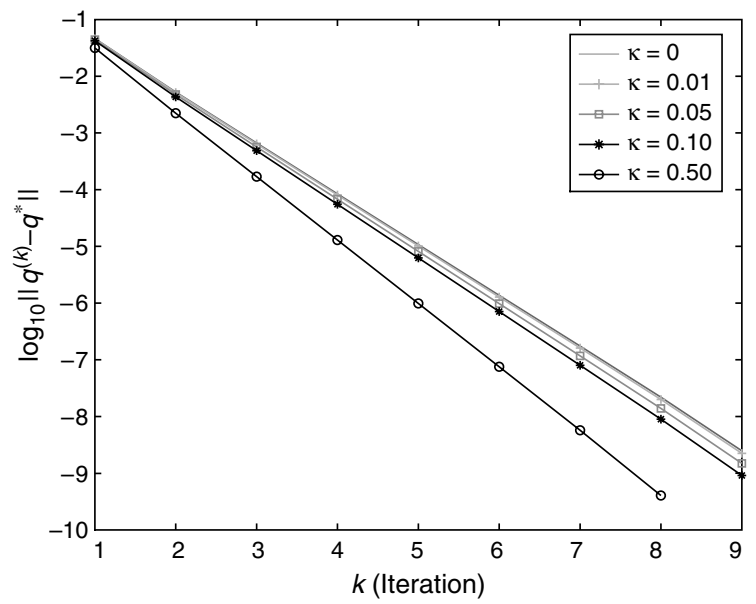

(a) Convergence of $\mathbf{q}^{(k)}$

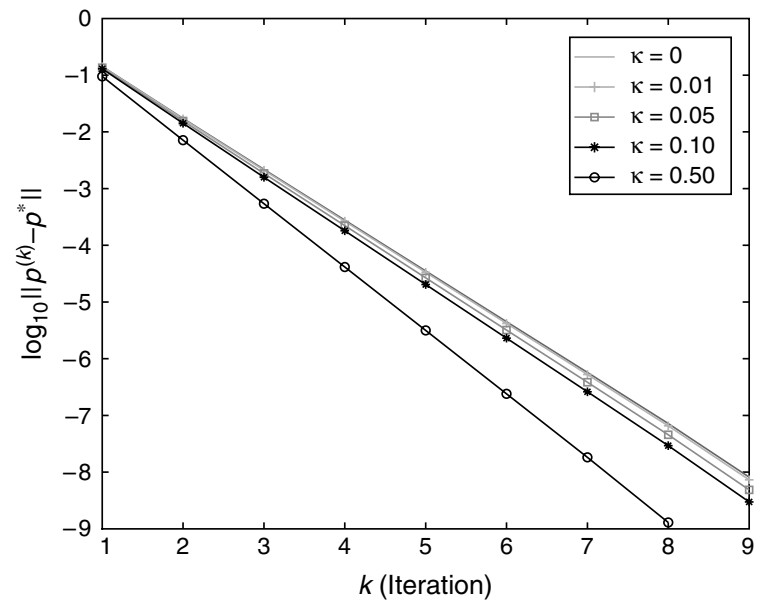

(b) Convergence of $\mathbf{p}^{(k)}$

Notes. $d_{i}(\mathbf{p})=\left(\alpha_{i}-\beta_{i} p_{i}\right) /\left(\sum_{j=1}^{n}\left(\alpha_{j}-\beta_{j} p_{j}\right)+\kappa\right)$ where $\beta=(0.1,0.3,0.5$, $0.7,0.9)$ and the ratios $\alpha_{i} /\left(\beta_{i} \cdot c_{i}(1)\right)$ 's are given by $(1.01,1.02,1.03$, $1.04,1.05)$. Initialization $\mathbf{p}^{(0)}=\left(C_{1}^{\prime}(0), \ldots, C_{n}^{\prime}(0)\right) . n=5$. 
count for each value of $\kappa$. It shows linear convergence as predicted by Proposition 9. The convergence rate of the price vector is also linear, as illustrated by Figure 1 . This figure indicates that the number of required iterations is smaller when there are more competing firms.

We now vary the number of firms in competition and use $n=2,5,10$. For each $n$, the set of $\lambda_{i}$ values and $y_{i}$ values is given in Table 4 . We fix $\kappa$ at 0.01 . We obtain similar linear convergence results, shown in Figure 2.

Additional tests have been carried out for the Cobb-Douglas and linear attraction functions. See Figures 3 and 4 .

\section{Appendix}

\section{A.1. Continuity of $D_{i}(\delta)$}

By the continuity assumptions on all involved functions, $f_{i}\left(x_{i}, \delta\right):=L_{i}\left(x_{i} \mid \delta\right)-R_{i}\left(x_{i} \mid \delta\right)$ is continuous in $\left(x_{i}, \delta\right)$. For each $\delta \in \Delta$, there is a unique $D_{i}(\delta)$ satisfying $f_{i}\left(D_{i}(\delta), \delta\right)=$ 0 by Proposition 3. Furthermore, the argument following Proposition 3 shows $D_{i}(\delta)$ is strictly increasing in $\delta$.

Consider any sequence $\delta_{k} \rightarrow \delta_{0}$. If $\lim _{k \rightarrow \infty} D_{i}\left(\delta_{k}\right)$ exists, then by the continuity of $f$, we have

$$
\begin{aligned}
0 & =\lim _{k \rightarrow \infty} f\left(D_{i}\left(\delta_{k}\right), \delta_{k}\right)=f\left(\lim _{k \rightarrow \infty} D_{i}\left(\delta_{k}\right), \lim _{k \rightarrow \infty} \delta_{k}\right) \\
& =f\left(\lim _{k \rightarrow \infty} D_{i}\left(\delta_{k}\right), \delta_{0}\right),
\end{aligned}
$$

implying $\lim _{k \rightarrow \infty} D_{i}\left(\delta_{k}\right)=D_{i}\left(\delta_{0}\right)$. Assuming $\lim _{k \rightarrow \infty} D_{i}\left(\delta_{k}\right)$ does not exist, apply the above argument to subsequences converging to $\limsup _{k \rightarrow \infty} D_{i}\left(\delta_{k}\right)$ and $\liminf _{k \rightarrow \infty} D_{i}\left(\delta_{k}\right)$, respectively. Then, their limits should be the same. Thus, $D_{i}(\delta)$ is continuous in $\delta$.

\section{A.2. Existence and Monotonicity of $\psi_{i}$}

Because $a_{i}(\cdot)$ is one to one and $\delta=q_{i}+\hat{q}_{i}$, condition (6) is equivalent to (8). Because $c_{i}(\cdot)$ is nondecreasing and $a_{i}^{-1}(\cdot)$ is strictly increasing, the left side of (6) increases strictly. Because $\eta_{i} \circ a_{i}^{-1}(\cdot)$ is nonincreasing and $1+(\cdot) /\left(\hat{q}_{i}+\kappa\right)$ is strictly increasing, the right side of (6) decreases strictly. As $q_{i}$ approaches 0 and $a_{i}(0)$, both limits are the same as those in the proof of Proposition 3. Thus $\psi_{i}(\cdot)$ is well defined.

Now the lifting-and-dropping argument following Proposition 3 can be applied to prove Proposition 7, which shows the monotonicity of $\psi_{i}(\cdot)$.

\section{References}

Allon, G., A. Federgruen. 2004. Service competition with general queueing facilities. Working paper.
Anderson, S. P., A. De Palma, J.-F. Thisse. 1996. Discrete Choice Theory of Product Differentiation. MIT Press, Cambridge, MA.

Benassy, J.-P. 1989. Market size and substitutability in imperfect competition: A Bertrand-Edgeworth-Chamberlin model. Rev. Econom. Stud. 56 217-234.

Bernstein, F., A. Federgruen. 2003. Pricing and replenishment strategies in a distribution system with competing retailers. Oper. Res. 51(3) 409-426.

Bernstein, F., A. Federgruen. 2004a. Comparative statics, strategic complements and substitutes in oligopolies. J. Math. Econom. 40(6) 713-746.

Bernstein, F., A. Federgruen. 2004b. A general equilibrium model for industries with price and service competition. Oper. Res. 52(6) 868-886.

Bernstein, F., F. Chen, A. Federgruen. 2002. Vendor managed inventories and supply chain coordination: The case with one supplier and competing retailers. Working paper.

Bertrand, J. 1883. Théorie mathématique de la richesse sociale. J. Savants 499-508.

Bertsekas, D. P. 1995. Nonlinear Programming. Athena Scientific.

Besanko, D., S. Gupta, D. Jain. 1998. Logit demand estimation under competitive pricing behavior: An equilibrium framework. Management Sci. 44 1533-1547.

Cachon, G. P., P. T. Harker. 2002. Competition and outsourcing with scale economies. Management Sci. 48(10) 1314-1333.

Caplin, A., B. Nalebuff. 1991. Aggregation and imperfection competition: On the existence of equilibrium. Econometrica 59 25-59.

Chamberlin, E. H. 1933. The Theory of Monopolistic Competition. Harvard University Press, Boston, MA.

Cournot, A. 1838. Recherches sur les Principes Mathématiques de la Théorie des Richesses. Macmillan. English ed.: N. Bacon, ed. 1863. Researches into the Mathematical Principles of the Theory of Wealth.

Debreu, G. 1960. Book review of Individual Choice Behavior: A Theoretical Analysis. The Amer. Econom. Rev. 50 188-190.

Dubey, P., O. Haimanko, A. Zapechelnyuk. 2003. Strategic complements and substitutes, and potential games. Working paper.

Edgeworth, F. 1922. The mathematical economies of Professor Amoroso. Econom. J. 32 400-407.

Edgeworth, F. 1925. The pure theory of monopoly. Papers Relating to Political Economy 111-142.

Friedman, J. 1983. Oligopoly Theory. Cambridge University Press.

Hotelling, H. 1929. Stability in competition. Econom. J. 39 41-57.

Luce, R. D. 1959. Individual Choice Behavior: A Theoretical Analysis. Wiley.

Mahajan, S., G. van Ryzin. 1998. Supply chain contracting and coordination with stochastic demand. S. Tayur, M. Magazine, R. Ganeshan, eds. Quantitive Models for Supply Chain Management. Kluwer.

Medhi, J. 2003. Stochastic Models in Queueing Theory, 2nd ed. Academic Press.

Milgrom, P., J. Roberts. 1990. Rationalizability, learning, and equilibrium in games with strategic complementarities. Econometrica 58 1255-1277.

Milgrom, P., C. Shannon. 1994. Monotone comparative statics. Econometrica 62 157-180.

Mizuno, T. 2003. On the existence of a unique price equilibrium for models of product differentiation. Internat. J. Indust. Organ. 62 761-793. 
Roberts, J., H. Sonnenschein. 1977. On the foundations of the theory of monopolistic competition. Econometrica 45 101-113.

Robinson, J. 1933. The Economics of Imperfect Competition. Macmillan.

So, K. C. 2000. Price and time competition for service delivery. Manufacturing Service Oper. Management 2 392-407.

Topkis, D. M. 1979. Equilibrium points in nonzero-sum n-person submodular games. SIAM J. Control Optim. 17 773-787.
Vives, X. 1985. On the efficiency of Bertrand and Cournot equilibria with product differentiation. J. Econom. Theory 36 $166-175$.

Vives, X. 1990. Nash equilibrium with strategic complementarities. J. Math. Econom. 305-321.

Vives, X. 1999. Oligopoly Pricing: Old Ideas and New Tools. MIT Press, Cambridge, MA. 